\title{
Formation of the largest galactic cores through binary scouring and gravitational wave recoil
}

\author{
Imran Tariq Nasim, ${ }^{1 \star}$ Alessia Gualandris, ${ }^{1}$ Justin I. Read ${ }^{\circledR},{ }^{1 \star}$ Fabio Antonini ${ }^{\circledR},{ }^{1,2}$ Walter Dehnen ${ }^{\circledR 3,4,5 \star}$ \\ and Maxime Delorme ${ }^{1,6}$ \\ ${ }^{1}$ Department of Physics, University of Surrey, Guildford GU2 7XH, Surrey, UK \\ ${ }^{2}$ School of Physics and Astronomy, Cardiff University, Cardiff CF24 3AA, UK \\ ${ }^{3}$ Astronomisches Rechen-Institut, Zentrum für Astronomie der Universität Heidelberg, M"onchhofstr 12-14, D-69120 Heidelberg, Germany \\ ${ }^{4}$ Universitäts-Sternwarte München, Scheinerstr 1, D-81679 Munich, Germany \\ ${ }^{5}$ School of Phyiscs and Astronomy, University of Leicester, University Rd, Leicester LE1 7RH, UK \\ ${ }^{6}$ Département d'Astrophysique/AIM, CEA/IRFU, CNRS/INSU, Université Paris-Saclay, Université de Paris, F-91191 Gif-sur-Yvette, France
}

Accepted 2021 February 11. Received 2021 January 15; in original form 2020 October 27

\begin{abstract}
Massive elliptical galaxies are typically observed to have central cores in their projected radial light profiles. Such cores have long been thought to form through 'binary scouring' as supermassive black holes (SMBHs), brought in through mergers, form a hard binary and eject stars from the galactic centre. However, the most massive cores, like the $\sim 3 \mathrm{kpc}$ core in A2261-BCG, remain challenging to explain in this way. In this paper, we run a suite of dry galaxy merger simulations to explore three different scenarios for central core formation in massive elliptical galaxies: 'binary scouring', 'tidal deposition', and 'gravitational wave $(\mathrm{GW})$ induced recoil'. Using the GRIFFIN code, we self-consistently model the stars, dark matter, and SMBHs in our merging galaxies, following the SMBH dynamics through to the formation of a hard binary. We find that we can only explain the large surface brightness core of A2261-BCG with a combination of a major merger that produces a small $\sim 1 \mathrm{kpc}$ core through binary scouring, followed by the subsequent GW recoil of its SMBH that acts to grow the core size. Key predictions of this scenario are an offset SMBH surrounded by a compact cluster of bound stars and a non-divergent central density profile. We show that the bright 'knots' observed in the core region of A2261-BCG are best explained as stalled perturbers resulting from minor mergers, though the brightest may also represent ejected SMBHs surrounded by a stellar cloak of bound stars.
\end{abstract}

Key words: black hole physics - gravitational waves - methods: numerical-galaxies: interactions-galaxies: kinematics and dynamics-galaxies: nuclei .

\section{INTRODUCTION}

Observations by the Hubble Space Telescope (HST) have revealed a clear bimodality in the population of elliptical galaxies, with brighter ellipticals $\left(M_{B} \leq-21\right)$ tending to have 'cored' central surface brightness profiles and fainter ellipticals $\left(M_{B}>-21\right)$ showing 'cuspy' central profiles instead (King \& Minkowski 1966; Faber et al. 1997; Graham 2019). While cored galaxies tend to have a small degree of rotation with boxlike isophotes, cuspy galaxies have discy isophotes with a faster rotation (Kormendy \& Bender 1996; Lauer et al. 2007). Krajnović et al. (2013) show that the majority of massive, slowly rotating galaxies have surface brightness cores and that all slow rotating galaxies with stellar masses above $2 \times 10^{11} \mathrm{M}_{\odot}$ have such cores. This suggests that the observed structural differences arise due to different formation pathways of elliptical galaxies.

In the galactic merger scheme presented by Naab \& Ostriker (2017), massive elliptical galaxies, which are generally gas-poor, form through a multistage process where the early evolution is dominated by in situ star formation accompanied by multiple accretion

^E-mail: i.nasim@surrey.ac.uk (ITN); j.read@surrey.ac.uk (JIR);

wdehnen64@gmail.com (WD) events with star-bursting progenitors. Subsequent growth $(z \leq 3)$ is dominated by dry (gas-poor) mergers, which has been shown to rapidly grow the size of ellipticals (Naab, Johansson \& Ostriker 2009). Fainter ellipticals, on the other hand, form through in situ star formation along with multiple accretion events with gas-rich disc galaxies leading to steeply rising central surface brightness profiles (Naab \& Trujillo 2006; Johansson, Naab \& Burkert 2009). While this framework explains many observed properties, the existence of surface brightness cores in the most massive ellipticals poses a challenging theoretical problem. If massive ellipticals form from the merger of fainter cuspy galaxies (Feldmann, Carollo \& Mayer 2011; Moster, Naab \& White 2013), they should exhibit cuspy profiles, as it has been shown that mergers preserve the steep density cusps of the merging galaxies (e.g. Boylan-Kolchin, Ma \& Quataert 2004; Dehnen 2005).

Currently the most accepted mechanism for core formation in ellipticals is scouring by binary supermassive black holes (SMBHs) (Begelman, Blandford \& Rees 1980; Hills 1983; Quinlan 1996). During the merger of two gas-poor galaxies, the central SMBHs sink to the centre of the remnant due to dynamical friction (Chandrasekhar 1943) until they form a gravitationally bound pair. The massive black hole binary (BHB) interacts with the surrounding stellar environment, causing the ejection of stars on intersecting orbits. 
This removes energy and angular momentum from the binary, causing it to harden (Hills 1983; Quinlan 1996; Sesana, Haardt \& Madau 2006), and carves a core in the stellar distribution. If the binary shrinks to a separation, typically of the order of milliparsecs, where emission of gravitational waves (GWs) becomes important, the evolution proceeds quickly to inspiral and coalescence to a single SMBH. Anisotropic emission of GWs imparts a kick velocity, known as GW recoil, to the newly formed black hole. Due to the recent advancements in numerical relativity (Campanelli et al. 2006; Lousto \& Zlochower 2011; Healy, Lousto \& Zlochower 2014; Healy et al. 2018), it has become possible to simulate the merger of SMBHs in full general relativity and evaluate the size of GW kicks. Interestingly, recoil kicks range from a few hundred to $\sim 5000 \mathrm{~km} \mathrm{~s}^{-1}$, depending on the configuration of the merging SMBHs and the relative orientation of their spins (Campanelli et al. 2007; González et al. 2007; Lousto \& Zlochower 2011; Lousto \& Healy 2019). Large GW recoils remove the SMBHs from the central region, leading to damped oscillatory motion with repeated passages through the core. During such passages the SMBH injects energy into the central region, enlarging the core formed during the phase of binary hardening (e.g. Boylan-Kolchin et al. 2004; Gualandris \& Merritt 2008; Merritt, Schnittman \& Komossa 2009). The oscillations are slowly damped by dynamical friction until the SMBH reaches dynamical equilibrium with the background stellar distribution (Gualandris \& Merritt 2008). Because dynamical friction is inefficient in flat density profiles (e.g. Read et al. 2006), the oscillatory motion is long lived, and the core continues to be enlarged at each oscillation. While $\mathrm{GW}$ recoil can in principle lead to the ejection of the SMBH and its associated bound star cluster from the host galaxy (Merritt et al. 2009), recoils exceeding the central escape speed (typically $\sim 1000 \mathrm{kms}^{-1}$ for massive galaxies; Devecchi et al. 2009) are expected to be rare (Blecha et al. 2016). A study by Lousto et al. (2012) showed the probability of recoil velocities exceeding the escape velocity is $\sim 5$ per cent for galaxies with escape velocities of $1000 \mathrm{kms}^{-1}$ and $\sim 20$ per cent for galaxies with escape velocities of $500 \mathrm{kms}^{-1}$

Alternative processes for core formation include the 'Multiple SMBH scouring' mechanism by Kulkarni \& Loeb (2012), a natural extension of the core-scouring mechanism to multiple SMBHs, the 'SMBH-AGN' scenario by Martizzi, Teyssier \& Moore (2012) in which a core forms through repeated fluctuations of the inner gravitational potential on a time-scale comparable to the local dynamical time, ${ }^{1}$ and the 'stalled perturber' scenario (Read et al. 2006; Goerdt et al. 2010) where a sinking satellite transfers energy to the background galaxy causing a reduction in central density and the formation of a constant density core, with the satellite stalling at the edge of the core. In practice, all of these mechanisms could act in tandem to a greater or lesser degree to drive the observed cores in massive ellipticals.

Alongside the above theoretical uncertainties in the physics of core formation in ellipticals, there have also been observational challenges. In particular, determining the size of the core has proven to be a non-trivial task. The light profiles of ellipticals are well

\footnotetext{
${ }^{1}$ Such a mechanism has been discussed extensively in the literature where the potential fluctuations owe to gas flows driven by stellar feedback (e.g. Read \& Gilmore 2005; Pontzen \& Governato 2012; Read, Walker \& Steger 2019). Here, gas flows are driven by AGN feedback instead. This scenario may not be able to produced large - kpc scale - cores but if possible such a large-scale feedback will clear the inner galaxy of gas and hence prevent subsequent AGN activity.
}

described by the 3-parameter Sérsic profile (Sérsic 1963, 1968) over a large radial range. The most luminous ellipticals, however, show a departure from the Sérsic law in their central regions, at a radius widely known as the 'break' or 'core' radius. In these galaxies, the profiles break downward from the inward extrapolation of the outer Sérsic law. Initially the core size of a galaxy was determined by fitting the so-called 'Nuker-profile' (Lauer et al. 1995) to the surface brightness profile, a method that however depends sensitively on the radial fitting range and yields unreliable results when fit to surface brightness profiles with a large radial extent (e.g. Graham et al. 2003; Dullo \& Graham 2012). In more recent years, it has become customary to incorporate a central flattening in the light profile by adopting a 6-parameter core-Sérsic profile (Graham et al. 2003; Trujillo et al. 2004) which provides a reliable measurement of the core size even over a large radial range (e.g. Dullo \& Graham 2012, 2013, 2014). Furthermore, it has been shown that adopting a multicomponent model rather than a single core-Sérsic model over the entire radial range provides a more reliable estimate of the core size (Dullo \& Graham 2014; Dullo 2019). Measured core sizes for massive ellipticals - derived in this way - are typically tens to a few hundred parsecs (e.g. Dullo \& Graham 2014), while cores larger than $1 \mathrm{kpc}$ are rare. A study by Lauer et al. (2007) considered a large sample of brightest cluster galaxies (BCGs) and found that fewer than 10 systems had a core size of $\sim 1 \mathrm{kpc}$ or greater, with the largest cored system being NGC 6166 which has a core size of $\sim 1.5 \mathrm{kpc}$. More recently, Dullo (2019) considered the largest sample of 'largecore' galaxies to date, finding that only 13(7) galaxies have core sizes larger than $0.5(1) \mathrm{kpc}$.

Large-core galaxies are found to obey the same scaling relations between break radius and surface brightness or spheroid luminosity as normal core galaxies, as well as a log-linear break radiusSMBH mass relation, when a measurement is available (Dullo 2019). However, directly measured SMBHs are overmassive with respect to predictions from the $M_{\mathrm{SMBH}}-L$ and the $M_{\mathrm{SMBH}}-\sigma$ relations, with a more significant offset for the latter relation. This is generally interpreted as a result of an increased number of dry mergers for the most massive galaxies (e.g. Dullo 2019).

An extremely large surface brightness core has been found in the BCG of Abell 2261 (A2261-BCG hereafter) (Postman et al. 2012). Assuming a single-component model, Bonfini \& Graham (2016) infer a core-Sérsic break radius of $3.6 \mathrm{kpc}$, while assuming a multicomponent model Dullo (2019) derive a core radius of $2.7 \mathrm{kpc}$. With either estimate, this represents the second largest depleted core known to date (Dullo 2019). The earlier measurement, in particular, is so extreme to imply an unrealistically large number of dry major mergers, at least in the context of the standard Lambda cold dark matter $(\Lambda \mathrm{CDM})$ cosmology (Li et al. 2007; Bonfini \& Graham 2016). Another peculiarity of A2261-BCG is that it contains a number of objects in the core region referred to as 'knots', as they appear as high-density regions in the surface brightness profile (Postman et al. 2012; Bonfini \& Graham 2016). Dosopoulou \& Antonini (2017) suggested that these knots could be stalled satellites that decayed to the centre of the main galaxy through dynamical friction. Together with the extremely large core size, the knots make this galaxy a unique system to study core formation scenarios.

In this paper, we present a detailed numerical study of the effect of successive galactic dry mergers, both major and minor, on the core-size of ellipticals. Each merging galaxy hosts an SMBH and we model the mergers using the new GRIFFIN Fast Multiple Method (Dehnen 2002, 2014). This allows us to simultaneously capture the $\mathrm{N}$-body dynamics of the merger and the SMBH-star and SMBH- 
SMBH interactions up to and after the formation of a hard binary (Nasim et al. 2020). We select a sequence of dry mergers consistent with expectations in $\Lambda \mathrm{CDM}$ and representative of A2261-BCG. For a subset of our simulations, we additionally model the impact of the GW recoil of the newly formed SMBH following binary coalescence. Our key finding is that three distinct mechanisms are involved in the formation of the very largest surface brightness cores in ellipticals. The initial core is formed by binary scouring during the first dry major merger and is only minimally enlarged by subsequent major mergers. GW recoil, on the other hand, can deplete the core further by ejecting additional stellar mass. Finally, minor mergers are responsible for the tidal deposition of material, including small satellites, on the edge the core. This further expands the size of the core and could plausibly explain the 'knots' observed in A2261-BCG. We note that these cores are identified and measured by fitting core-Sérsic models to the surface density profiles, in analogy with the approach most commonly adopted by observers (e.g. Graham 2019). The presence of a core, i.e. a region of (nearly) constant density, in the surface density profile of a galaxy does not imply a core in the three-dimensional density profile. Our models instead produce a shallow cusp in spatial density.

This article is organized as follows. The numerical simulations and adopted galactic models are presented in Section 2. The role of the first major merger is investigated in Section 3, followed by a description of subsequent mergers combined with an analysis of core sizes and mass deficits in Section 4. GW recoil is studied in Section 5, where additional simulations of kicked SMBHs are presented. Our results on the relative importance and effects of the different core formation mechanisms are discussed in Section 6. Finally, in Section 7, we present our conclusions on how we can best-explain the extremely large core observed in A2261-BCG based on the simulations presented here.

\section{NUMERICAL SIMULATIONS}

\subsection{Initial conditions}

We consider multicomponent galaxy models consisting of a stellar bulge, dark matter (DM) halo, and a central massive black hole (BH). The bulge follows a Sérsic model (Sérsic 1963, 1968) in projection

$I(R)=I_{0} \mathrm{e}^{-b\left(R / R_{\mathrm{e}}\right)^{1 / n}}$,

where $I_{0}$ is the central intensity, $R_{\mathrm{e}}$ is the (projected) effective halflight radius, $n$ describes the curvature of the outer profile, and $b$ is a function of $n$ given by $b=2 n-1 / 3+0.009876 / n$ (Terzić \& Graham 2005). The corresponding space density profile is well approximated by (Terzić \& Graham 2005)

$$
\begin{aligned}
& \rho(r)=\rho_{0}\left(\frac{r}{R_{\mathrm{e}}}\right)^{-p} \mathrm{e}^{-b\left(r / R_{\mathrm{e}}\right)^{1 / n}} \\
& \rho_{0}=\Upsilon I_{0} b^{n(1-p)} \frac{\Gamma(2 n)}{2 R_{\mathrm{e}} \Gamma(n(3-p))},
\end{aligned}
$$

where $p=1.0-0.6097 / n+0.05563 / n^{2}$ (Márquez et al. 2000), $\Gamma$ is the gamma function and for the mass-to-light ratio we assume $\Upsilon=3.5 \mathrm{M}_{\odot} / \mathrm{L}_{\odot}($ Bonfini \& Graham 2016).

The dark matter halo follows a Navarro, Frenk \& White (1996) profile (NFW)

$$
\rho(r)=\frac{\rho_{0}}{(r / a)(1+r / a)^{2}},
$$

which is described by the two free parameters: $a$ and $\rho_{0}$. The scale length parameter $a$ is set equal to the scale radius $r_{\mathrm{s}}=r_{200} / c$, where $c$ is a concentration parameter and $r_{200}$ is the distance from the
Table 1. The core-Sérsic fit parameters (see equation 11) for A2261-BCG from (Bonfini \& Graham 2016) (B16) and (Dullo 2019) (D19).

\begin{tabular}{lcccccc}
\hline Study & $\begin{array}{c}\mu_{b} \\
{\left[\mathrm{mag} \mathrm{arcsec}^{-2}\right]}\end{array}$ & $n$ & $\alpha$ & $\gamma$ & $\begin{array}{c}R_{e} \\
{[\mathrm{kpc}]}\end{array}$ & $\begin{array}{c}R_{b} \\
{[\mathrm{kpc}]}\end{array}$ \\
\hline B16 & 19.59 & 3.9 & 3.6 & 0.02 & 38.86 & 3.63 \\
D19 & 18.69 & 2.1 & 5.0 & 0.0 & 17.6 & 2.71 \\
\hline
\end{tabular}

centre of the halo where the mean enclosed density is 200 times greater than the critical density of the Universe at redshift $z=0$, $\rho_{\mathrm{c}}=136.05 \mathrm{M}_{\odot} \mathrm{kpc}^{-3}$. We adopt the relation derived by Dutton \& Macciò (2014) to set the concentration parameter. The parameter $\rho_{0}$ is set by the mass enclosed within a sphere of radius the $r_{200}$, which is given by $M_{200}=200 \rho_{c} \frac{4}{3} \pi r_{200}^{3}$. The enclosed mass profile for equation (3) is given by

$M(r)=4 \pi \rho_{0} a^{3}\left[\ln (1+r / a)-\frac{r / a}{1+r / a}\right]$.

Because the NFW mass profile diverges logarithmically with radius, we impose an outer cut-off at $r_{200}$ with a logarithmic slope of -5 .

We use the AGAMA action-based modelling library (Vasiliev 2019) to set up self-consistent initial conditions for our spherically symmetric, multicomponent, isotropic models. To this aim, we considered the combined potential of the stellar bulge, the dark matter halo, and the central SMBH, which is represented as a point mass $M_{\bullet}$ at rest at the origin. We then sampled each component from their distribution function to produce the multicomponent models.

\subsection{Merger set-up}

In order to investigate the origin of the largest observed cores, we model mergers of massive elliptical galaxies including major and minor mergers and consider different sequences of such mergers. We take A2261-BCG as our fiducial galaxy with an extremely large core, whose structural parameters were derived first by Postman et al. (2012) and Bonfini \& Graham (2016), and more recently by Dullo (2019) by fitting a core-Sérsic model (see Table 1).

The initial conditions (ICs) of the progenitor galaxies are listed in Table 2. IC-1-IC-3 represent the progenitor galaxies for the initial 1:1 major merger, adopting the parameters from B16, and differ only by the number of star and DM particles used in the $N$-body realizations. IC-1 is the progenitor at the fiducial resolution, IC-2 has the same stellar resolution as IC-1 but has 10 times more dark matter particles, and IC-3 has the same dark matter resolution as IC- 1 but with 10 times more star particles. The stellar mass is set by assuming that the observed mass is already present in the progenitor galaxies of the initial major merger. The effective radius is set by assuming that it increases by a factor of 2 in a dry equal mass merger (Naab et al. 2009), as a consequence of the virial theorem. IC-4IC-6 correspond to the progenitor galaxies for the subsequent minor and major mergers: in IC-4 the structural parameters are obtained via abundance matching (Behroozi, Wechsler \& Conroy 2013) assuming an effective radius $R_{\mathrm{e}}=0.015 r_{200}$ (Kravtsov 2013), IC-5 is a scaled down model of IC- 1 by a factor of 5 and IC- 6 a scaled down model of IC- 1 by a factor of 2. IC-6-IC-8 represent the progenitor galaxies for the initial 1:1 major merger assuming the parameters by D19: IC-7 adopts the SMBH mass from B 16 and IC- 8 adopts the SMBH mass from D19. The merger parameters are given in Table 3. The merger remnants $\mathrm{A} 1-\mathrm{A} 3$ are from the initial equal mass major merger with different resolutions. Merger remnants B1-B4 correspond to 
Table 2. Properties of the progenitor galaxies. From left to right, the columns give stellar mass $\left(M_{\star}\right)$, dark matter halo mass $\left(M_{\mathrm{DM}}\right)$, central SMBH mass $\left(M_{\bullet}\right)$, effective radius $\left(R_{\mathrm{e}}\right)$, Sérsic index $(n)$, number of star particles $\left(N_{*}\right)$, and number of DM particles $\left(N_{\mathrm{DM}}\right)$ in the realizations, brief progenitor description.

\begin{tabular}{lcccccccl}
\hline Progenitor & $\begin{array}{c}M_{\star} \\
{\left[10^{10} \mathrm{M}_{\odot}\right]}\end{array}$ & $\begin{array}{c}M_{\mathrm{DM}} \\
{\left[10^{10} \mathrm{M}_{\odot}\right]}\end{array}$ & $\begin{array}{c}M_{\bullet} \\
{\left[10^{10} \mathrm{M}_{\odot}\right]}\end{array}$ & $\begin{array}{c}R_{\mathrm{e}} \\
{[\mathrm{kpc}]}\end{array}$ & $n$ & $\begin{array}{c}N_{*} \\
{\left[10^{6}\right]}\end{array}$ & $\begin{array}{c}N_{\mathrm{DM}} \\
{\left[10^{6}\right]}\end{array}$ & Description \\
\hline IC-1 & $2.2 \times 10^{2}$ & $1.0 \times 10^{4}$ & 1.27 & 19.43 & 3.9 & 1.0 & 1.0 & $1: 1$ merger progenitor using B16 parameters \\
IC-2 & $2.2 \times 10^{2}$ & $1.0 \times 10^{4}$ & 1.27 & 19.43 & 3.9 & 1.0 & 10.0 & Same as IC-1 with higher DM resolution \\
IC-3 & $2.2 \times 10^{2}$ & $1.0 \times 10^{4}$ & 1.27 & 19.43 & 3.9 & 10.0 & 1.0 & Same as IC-1 with higher stellar resolution \\
IC-4 & $1.6 \times 10^{1}$ & $2.0 \times 10^{3}$ & 0.101 & 4.41 & 4.0 & 1.0 & 1.0 & $1: 10$ merger progenitor (abundance matching) \\
IC-5 & $4.4 \times 10^{1}$ & $2.0 \times 10^{3}$ & 0.254 & 8.42 & 4.0 & 1.0 & 1.0 & $1: 10$ merger progenitor (scaled from IC-1) \\
IC-6 & $1.1 \times 10^{2}$ & $5.0 \times 10^{3}$ & 0.635 & 11.4 & 4.0 & 1.0 & 1.0 & $1: 4$ merger progenitor (scaled from IC-1) \\
IC-7 & $2.2 \times 10^{2}$ & $1.0 \times 10^{4}$ & 1.27 & 8.8 & 2.1 & 1.0 & 1.0 & $1: 1$ merger progenitor using D19 parameters \\
IC-8 & $2.2 \times 10^{2}$ & $1.0 \times 10^{4}$ & 3.23 & 8.8 & 2.1 & 1.0 & 1.0 & Same as IC-7 but with larger SMBH mass \\
IC-1a & $2.2 \times 10^{2}$ & $1.0 \times 10^{4}$ & 0 & 19.43 & 3.9 & 1.0 & 1.0 & Same as IC-1 but with no SMBH \\
IC-1aE & $2.2 \times 10^{2}$ & $1.0 \times 10^{4}$ & 0 & 19.43 & 3.9 & 1.0 & 45.0 & Same as IC-1 but with equal mass particles \\
IC-6a & $1.1 \times 10^{2}$ & $5.0 \times 10^{3}$ & 0 & 11.4 & 4.0 & 1.0 & 1.0 & Same as IC-6 but with no SMBH \\
\hline
\end{tabular}

Table 3. Merger parameters. Runs A1-3 correspond to the first equal-mass merger. Runs B1-4 are subsequent mergers from remnant A1. Run $\mathrm{C} 1$ is a subsequent 1:3 merger from B4. Run D1 is a merger simulation using the Sérsic parameters from D19 but with SMBH mass estimated from B16. Run D2 is a merger simulation using Sérsic parameters and SMBH mass derived from the $R_{b}-M_{\bullet}$ relation by D19. Columns from left to right: merger remnant label, primary $\left(M_{\bullet 1}\right)$ and secondary $\left(M_{\bullet 2}\right)$ galaxy SMBH mass, total dark-matter $\left(M_{\mathrm{DM}}\right)$ and stellar $\left(M_{\star}\right)$ mass, number of stellar $\left(N_{*}\right)$ and dark-matter $\left(N_{\mathrm{DM}}\right)$ particles, mass ratio of the galaxy merger $(q)$, initial separation $\left(r_{\mathrm{sep}}\right)$ and orbital eccentricity $(e)$ of the progenitor centres, labels of the progenitor models.

\begin{tabular}{lcccccccccc}
\hline Remnant & $\begin{array}{c}M_{\bullet} \\
{\left[10^{10} \mathrm{M}_{\odot}\right]}\end{array}$ & $\begin{array}{c}M_{\bullet 2} \\
{\left[10^{10} \mathrm{M}_{\odot}\right]}\end{array}$ & $\begin{array}{c}M_{\mathrm{DM}} \\
{\left[10^{10} \mathrm{M}_{\odot}\right]}\end{array}$ & $\begin{array}{c}M_{*} \\
{\left[10^{10} \mathrm{M}_{\odot}\right]}\end{array}$ & $\begin{array}{c}N_{*} \\
{\left[10^{6}\right]}\end{array}$ & $\begin{array}{c}N_{\mathrm{DM}} \\
{\left[10^{6}\right]}\end{array}$ & $q$ & $\begin{array}{c}r_{\text {sep }} \\
{[\mathrm{kpc}]}\end{array}$ & $e$ & Progenitors \\
\hline A1 & 1.27 & 1.27 & $2.0 \times 10^{4}$ & $4.4 \times 10^{2}$ & 2.0 & 2.0 & 1.0 & 39 & 0.95 & IC-1+ IC-1 \\
A2 & 1.27 & 1.27 & $2.0 \times 10^{4}$ & $4.4 \times 10^{2}$ & 2.0 & 20.0 & 1.0 & 39 & 0.95 & IC-2+ IC-2 \\
A3 & 1.27 & 1.27 & $2.0 \times 10^{4}$ & $4.4 \times 10^{2}$ & 20.0 & 2.0 & 1.0 & 39 & 0.95 & IC-3+ IC-3 \\
A1E & 0 & 0 & $2.0 \times 10^{4}$ & $4.4 \times 10^{2}$ & 2.0 & 90.0 & 1.0 & 39 & 0.95 & IC-1aE + IC-1aE \\
B1 & 2.54 & 0.101 & $2.2 \times 10^{4}$ & $4.6 \times 10^{2}$ & 3.0 & 3.0 & 0.1 & 45 & 0.95 & A1 + IC-4 \\
B2 & 2.54 & 0.254 & $2.2 \times 10^{4}$ & $4.8 \times 10^{2}$ & 3.0 & 3.0 & 0.1 & 45 & 0.95 & A1 + IC-5 \\
B3 & 2.54 & 0.635 & $2.5 \times 10^{4}$ & $5.5 \times 10^{2}$ & 3.0 & 3.0 & 0.25 & 45 & 0.95 & A1 + IC-6 \\
B4 & 2.54 & 1.27 & $3.0 \times 10^{4}$ & $6.6 \times 10^{2}$ & 3.0 & 3.0 & 0.5 & 45 & 0.95 & A1 + IC-1 \\
B3a & 2.54 & 0 & $2.5 \times 10^{4}$ & $5.5 \times 10^{2}$ & 3.0 & 3.0 & 0.25 & 45 & 0.95 & A1+ IC-6a \\
B4a & 2.54 & 0 & $3.0 \times 10^{4}$ & $6.6 \times 10^{2}$ & 3.0 & 3.0 & 0.5 & 45 & 0.95 & A1+ IC-1a \\
C1 & 3.81 & 1.27 & $4.0 \times 10^{4}$ & $8.8 \times 10^{2}$ & 4.0 & 4.0 & 0.33 & 45 & 0.95 & B4 + IC-1 \\
D1 & 1.27 & 1.27 & $2.0 \times 10^{4}$ & $4.4 \times 10^{2}$ & 2.0 & 2.0 & 1.0 & 20 & 0.95 & IC-7+ IC-7 \\
D2 & 3.23 & 3.23 & $2.0 \times 10^{4}$ & $4.4 \times 10^{2}$ & 2.0 & 2.0 & 1.0 & 20 & 0.95 & IC-8 + IC-8 \\
\hline
\end{tabular}

subsequent dry mergers after the initial merger of A1. B1 represents a 1:10 minor merger between the merger remnant A1 and IC-4, where the secondary galaxy has structural parameters determined from abundance matching (Behroozi et al. 2013). B2 represents a 1:10 minor merger between the merger remnant $\mathrm{A} 1$ and IC-5, where the secondary galaxy is a scaled down progenitor from A1. B3 represents a 1:4 minor merger between the merger remnant $\mathrm{A} 1$ and IC-6, where the secondary galaxy is scaled down from the remnant A1. B4 represents a 1:2 major merger between the merger remnant A1 and IC-1, where the secondary galaxy is the progenitor of the initial 1:1 major merger. $\mathrm{C} 1$ is the subsequent 1:3 major merger between remnant $\mathrm{B} 4$ and IC-1, where the secondary galaxy is the progenitor of the initial 1:1 major merger. D1 corresponds to the 1:1 initial major merger at the fiducial resolution using the Sérsic parameters from D19 but with the SMBH mass from B16. D2 corresponds to the 1:1 initial major merger at the fiducial resolution using the Sérsic parameters from D19 and the SMBH mass derived from the $R_{\mathrm{b}}-M$. from D19. Prior to all the subsequent mergers (B1-B4 and C1), we merge the binary black holes into a single $\mathrm{SMBH}$ located at the binary's centre of mass (COM): A1 for mergers B1-B4 and B4 for the merger $\mathrm{C} 1$.
For the initial 1:1 merger (A1-A3 and D1-D2) the galaxies were placed at a distance greater than $2 R_{\mathrm{e}}$ of the progenitor ${ }^{2}$ and on a bound elliptical orbit with eccentricity $e=0.95 .^{3}$ Such a large eccentricity was chosen to mimic merger conditions in cosmological simulations (e.g. Khochfar \& Burkert 2006) as well as to reduce computational time.

\subsection{Numerical method}

We perform all merger simulations with the code GRIFFIN (Dehnen 2014), which uses the Fast Multiple Method as force solver for gravity between stars and dark matter, using adaptive parameters

\footnotetext{
${ }^{2}$ We verified that this distance was sufficient by repeating mergers A1-A3 with separations of $100 \mathrm{kpc}$ and $250 \mathrm{kpc}$, and we observed no significant difference in the evolution.

${ }^{3}$ We define the eccentricity of the orbit by assuming that the galaxies are point masses. We note that this is an approximation due to the overlap between the progenitor galaxies.
} 
meant to avoid a tail of large force errors, with a mean relative force error of $3 \times 10^{-4}$. SMBH gravity is computed by direct summation and all trajectories are integrated using the leapfrog integrator. GRIFFIN has recently been shown to evolve BHBs in galactic mergers as accurately as direct summation methods (Nasim et al. 2020, 2021). For all of our numerical simulations, we adopt a softening length $\epsilon=23 \mathrm{pc}$ for the stars, dark matter particles, and the SMBHs. We verified that this choice of softening is appropriate by repeating merger A1 with a softening value $\epsilon=2.3 \mathrm{pc}$. The dynamical friction phase is unaffected, while the three-body phase shows a slightly reduced central surface brightness, though the difference is insignificant. We use a softening kernel that corresponds to smooth source particles with density proportional to $\left(r^{2}+\epsilon^{2}\right)^{-7 / 2}$ rather than the more commonly used Plummer softening which is characterized by a density proportional to $\left(r^{2}+\epsilon^{2}\right)^{-5 / 2}$. For a fixed value of $\epsilon$ the source mass is more concentrated than for the Plummer case, implying a weaker softening. The $\mathrm{SMBH}$ to star particle mass ratio for the initial conditions is in the range $5720 \leq M_{\star} / m_{\star} \leq 57200$, while the SMBH to DM particle ratio is in the range $127 \leq M_{\bullet} / m_{\mathrm{DM}}$ $\leq 1270$ (see Table 3), ensuring reduced stochastic effects in the evolution of the BHB (Nasim et al. 2020).

\section{INITIAL MERGER}

We start our analysis by investigating the effects of the first merger, i.e. the major mergers A1-A3 and D1-D2, focusing on the evolution of the BHB that forms and the formation of a core in the stellar density profile.

\subsection{Binary scouring}

When two progenitor galaxies merge, the SMBHs sink to the centre of the merger remnant via the process of dynamical friction (Chandrasekhar 1943) from the surrounding stellar and dark matter distribution. This process drives the SMBHs to a separation $a_{\mathrm{f}}$ at which the stellar mass $M_{\star}$ within the binary orbit is equal to twice the mass of the secondary black hole

$M_{\star}\left(r<a_{\mathrm{f}}\right)=2 M_{2}$.

The time when the binary reaches $a_{\mathrm{f}}$ roughly corresponds to the time when the SMBHs become gravitationally bound and is commonly taken to mark the transition from the dynamical friction dominated phase to the hardening dominated phase. This occurs at $t \approx 287 \mathrm{Myr}$ in remnants $\mathrm{A} 1-\mathrm{A} 3$ and $t \approx 80 \mathrm{Myr}$ in remnants $\mathrm{D} 1$ and $\mathrm{D} 2$, as can be seen in Figs 1 and 2 where we show the distance between the SMBHs as a function of time for the initial 1:1 major mergers (Fig. 1; simulations A1-A3 and D1-D2) and all subsequent mergers (Fig. 2; simulations B1-B2 and B3-C1).

Rapid hardening ensues as three-body encounters with stars become important and extract energy and angular momentum from the orbit, causing both a shrinking in the binary separation and ejection of stars to larger distances. As the central density is lowered and a core is a carved due to stellar ejections, this process is generally called binary scouring. The binary is formally considered hard at the hard binary separation $a_{\mathrm{h}}$, where the specific binding energy of the BHB exceeds the average specific kinetic energy of the stars (Merritt 2013)

$a_{\mathrm{h}}=\frac{G \mu}{4 \sigma^{2}}$

with $\mu=M_{1} M_{2} /\left(M_{1}+M_{2}\right)$ the reduced mass and $\sigma$ the line-of-sight velocity dispersion. An alternative definition which is better suited to $N$-body simulations is given by

$a_{\mathrm{h}}=\frac{\mu}{M_{\text {bin }}} \frac{r_{\mathrm{m}}}{4}=\frac{q}{(1+q)^{2}} \frac{r_{\mathrm{m}}}{4}$,

where $M_{\mathrm{bin}}$ is the mass of the BHB, $q=M_{2} / M_{1}$ is the black hole mass ratio, and $r_{\mathrm{m}}$ represents the radius containing a mass in stars equal to twice the mass of the primary black hole. We adopt this second definition, which for equal mass binaries simply gives $a_{\mathrm{h}}=r_{\mathrm{m}} / 16$.

We find $a_{\mathrm{f}}=0.75 \mathrm{kpc}$ and $a_{\mathrm{h}}=0.094 \mathrm{kpc}$ for runs A1-A3 and $a_{\mathrm{f}}=0.80,1.35 \mathrm{kpc}, a_{\mathrm{h}}=0.10,0.17 \mathrm{kpc}$ for runs D1 and D2, respectively. The larger values for the D1-D2 remnants may be surprising, considering that the progenitor galaxies have a smaller effective radius $R_{\mathrm{e}}$ (see Table 1). However, this can be attributed to the fact that the progenitor profiles in both D1-D2 have a significantly smaller Sérsic index than those in A1-A3, meaning they are less cuspy. The resulting lower central density can also be seen in the initial condition profiles in Fig. 3. In addition, the SMBH mass is larger in the D2 progenitor model, implying a larger radius of influence and hard binary separation. We run all of our merger simulations past the hard binary separation but end the simulation before the force softening length is reached. We show the surface luminosity profiles of models A1-A3 and D1-D2 in Fig. 3 (upper panels and lower middle/right panels, respectively). All profiles are computed with respect to the primary SMBH, which represents a viable centre even at early times ${ }^{4}$ As a reference, the vertical dotted lines in the figures indicate the three largest core radii known to date: IC 1101 (olive) with a $4.2 \mathrm{kpc}$ core, A2261-BCG (orange) with a $2.71 \mathrm{kpc}$ core, and $4 \mathrm{C}+74.13$ (purple) with a $2.24 \mathrm{kpc}$ (Dullo 2019).

The profiles of remnants A1-A3 show clear evidence of binary scouring: the central surface luminosity decreases during the binary hardening phase, between the end of the merger (marked roughly by the time when the separation $a_{\mathrm{f}}$ is reached) and the time when the binary reaches the hard-binary separation. This is due to the ejection of stars from the central region as a result of three-body interactions (e.g. Quinlan 1996; Milosavljević \& Merritt 2001; Merritt 2006). The ejected mass is deposited at larger distances but it is not clearly visible in the profiles as it is spread out over a large volume. No significant difference is found due to the adopted resolution in stellar and DM particles, as can be seen comparing models A2 and A3 with the fiducial resolution model A1.

We do not observe evidence of significant scouring in model D1 (See Fig. 3, bottom middle panel), while some scouring is present in model D2 (See Fig. 3, bottom right panel). This can be attributed to the flatter central profiles of the D1-D2 progenitors as compared to the A1-A3 progenitors, as a lower central density leads to reduced binary scouring (e.g. Rantala et al. 2018). In D2, this effect is, however, offset by its more massive SMBH, leading to significant scouring (see Fig. 3, bottom right panel).

To verify that binary scouring is the only mechanism responsible for the central core, we also ran an equal mass merger simulation without SMBHs (A1E). We adapted the resolution to have equal mass star and DM particles, which ensures there are no coring effects due to mass segregation..$^{5}$ The surface luminosity profile is given in Fig. 3 (bottom left panel), centred on the primary galaxy (Power et al. 2003)

\footnotetext{
${ }^{4} \mathrm{We}$ have verified that adopting different projections, as appropriate for merger remnants, yields no significant differences in the surface luminosity profiles.

${ }^{5}$ Mass segregation effects would be present at the fiducial resolution, leading to a small inner core due to the outward migration of lower mass particles. Such effects are not seen in the simulations with SMBHs (A1-A3) due to the effects of binary scouring.
} 

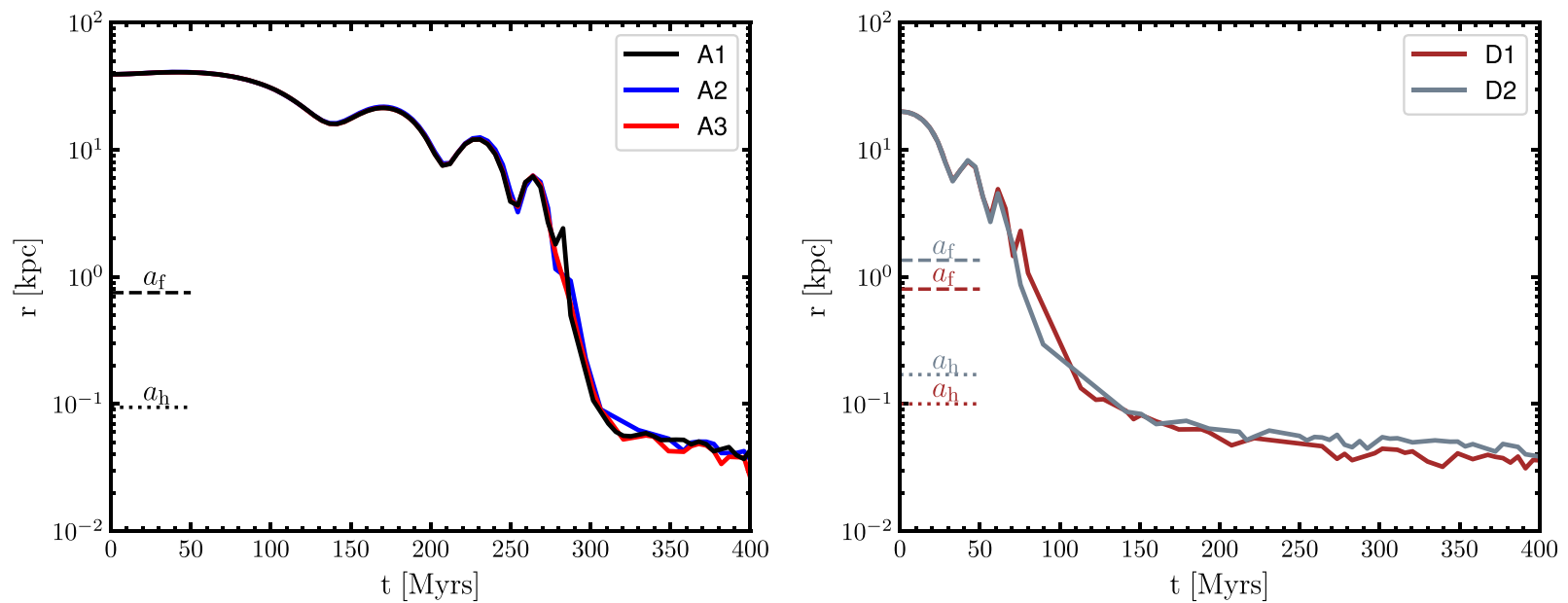

Figure 1. Distance between the SMBHs as a function of time for the 1:1 initial major mergers: A1-A3 (left-hand panel) and D1-D2 (right-hand panel). The horizontal dashed/dotted lines correspond to the $a_{\mathrm{f}} / a_{\mathrm{h}}$ separations.
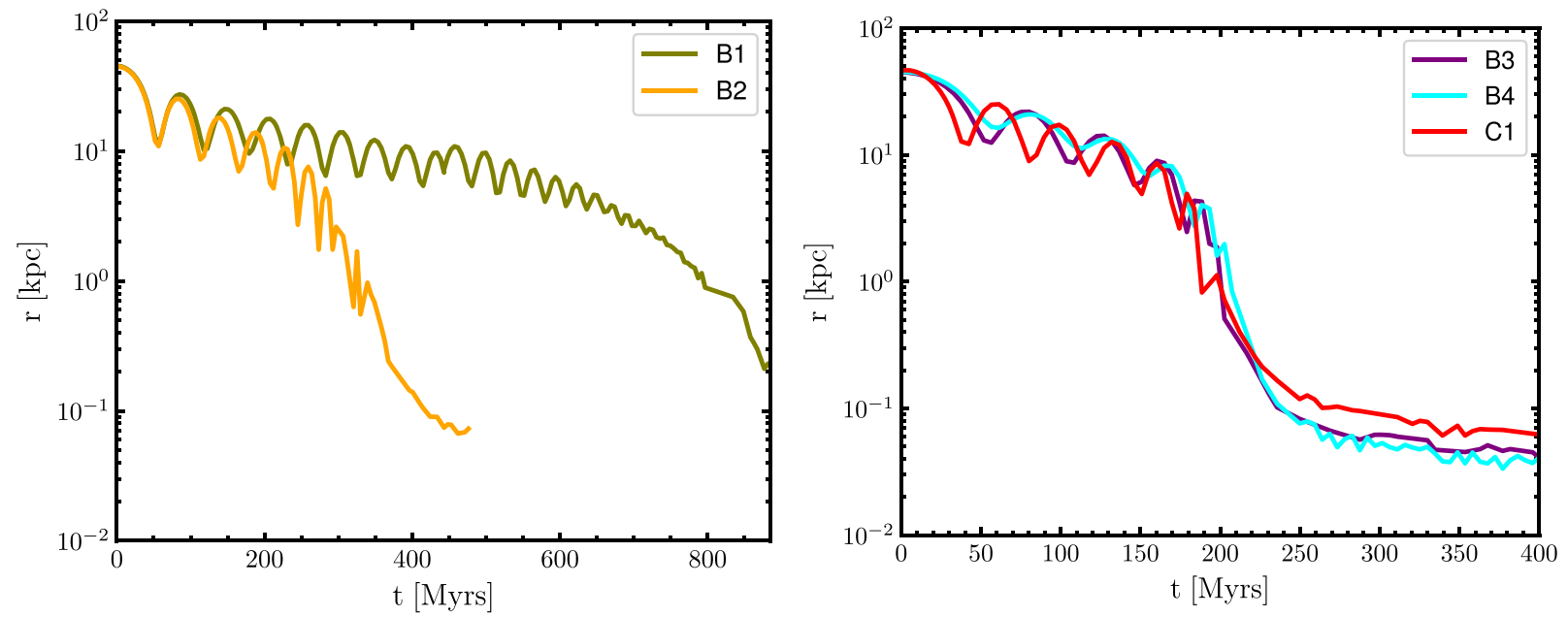

Figure 2. Distance between the SMBHs as a function of time for the subsequent mergers following the initial 1:1 major merger: B1-B2 (left-hand panel) and B3-C1 (right-hand panel).

and evaluated at the same times considered for the simulation with SMBHs (A1). As expected, no core is formed in the model without SMBHs.

We note that in this work we adopt the observational approach of defining a core as a flattening in the surface luminosity profile, measured by the break radius of the best-fitting core-Sérsic model (see Section 4.3). However, a core in the surface density profile does not necessarily imply a core in the spatial density profile; a shallow cuspy profile would also appear cored in projection. The spatial density profiles for the initial major mergers (shown in Fig. 4) reveal indeed the presence of a shallow cusp in all cases, scaling approximately as $\rho(r) \sim r^{-\gamma}$, with $0.5<\gamma<1$. As we will show in Section 5, cores produced by GW recoil of an SMBH are, however, of a different nature, and correspond to non-divergent profiles in spatial density.

\subsection{Violent relaxation}

The time evolving gravitational potential of a galactic major merger results in violent relaxation and dynamic mixing in which stars experience energy changes that are dependent upon their initial positions and velocities. This mixing operates on the time-scale of the merger itself, and is therefore important at early times. Evidence can be seen in the surface luminosity profiles of mergers D1 and D2 in the form of deposition of material at intermediate radii. A more modest effect is seen in merger A1, due to its higher stellar density.

The effects of binary scouring and violent relaxation can be seen also in the evolution of the Lagrangian radii. We consider four radii corresponding to the following values of enclosed stellar mass, centred on the primary SMBH:

$$
\begin{aligned}
& M_{\star}\left(r<r_{1}\right)=\frac{1}{5} M_{\bullet} \\
& M_{\star}\left(r<r_{2}\right)=M_{\bullet} \\
& M_{\star}\left(r<r_{3}\right)=5 M_{\bullet} \\
& M_{\star}\left(r<r_{4}\right)=\frac{1}{4} M_{\star} .
\end{aligned}
$$

The combined stellar mass of both progenitor galaxies is considered, so at times when the galaxies are well separated the radius $r_{4}$ approximates the half-mass radius of the progenitor during a 1:1 equal mass merger. The evolution of the Lagrangian radii is shown 

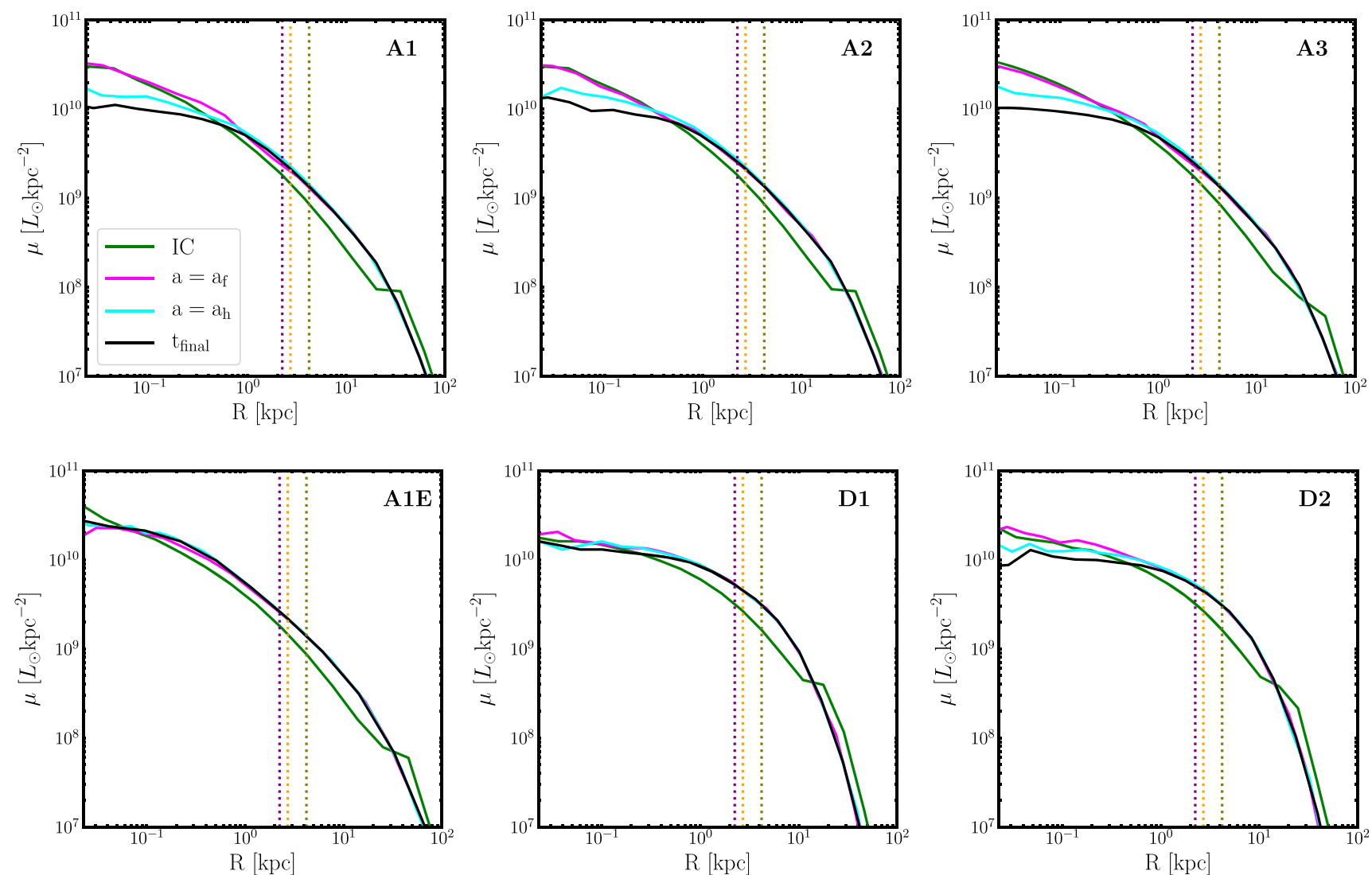

Figure 3. Surface luminosity profiles for the initial equal mass major mergers (A1-3, top panels), the equal mass major merger without SMBHs and with equal mass star and DM particles (A1E, bottom left panel), and the equal mass major mergers using D19 parameters: D1 and D2 (bottom left and bottom right panel, respectively). The profiles are computed at different times during the evolution: the initial condition (green line), the times when $a_{\mathrm{f}}$ (magenta) and $a_{\mathrm{h}}$ (cyan) are reached, and the end of the $\mathrm{N}$-body integration (black). The vertical dotted lines represent the three largest core radii known to date: IC 1101 (olive) with a $4.2 \mathrm{kpc}$ core, A2261-BCG (orange) with a $2.71 \mathrm{kpc}$ core, and $4 \mathrm{C}+74.13$ (purple) with a $2.24 \mathrm{kpc}$ (Dullo 2019). The innermost value of the radial range is set by the softening length of the stellar particles. Initial equal mass mergers with SMBHs show evidence of binary scouring as well as violent relaxation at larger radii, while no core is formed in the simulation without SMBHs. The surface mass densities can be computed by multiplying the surface luminosity by $\Upsilon=3.5 \mathrm{M}_{\odot} / \mathrm{L}_{\odot}$.

in Fig. 5 for the different models. In model A1, the Lagrangian radius $r_{4}$ decreases throughout the evolution, with the dips corresponding to the pericentre passages of the galaxies. A qualitatively similar behaviour is seen in models D1 and D2, but with a faster initial drop and less pronounced dips at pericentre passages due to the much faster inspiral of the secondary galaxy in these models. The innermost radius $r_{1}$ is approximately constant in model A 1 during the merger and increases during binary hardening, as a result of the stellar ejections from the binary. Binary scouring is most effective prior to the binary reaching $a_{\mathrm{h}}$, and then continues at a lower rate, as also seen in the simulations of Rantala et al. (2018). On the other hand, $r_{1}$ increases very slowly in the D1 remnant, supporting the earlier conclusion that binary scouring is inefficient in this model. The evolution of $r_{1}$ in model D2 is intermediate between that of models A1 and D1, again supporting the conclusion of a modest binary scouring mediated by the more massive central SMBH.

\section{SUBSEQUENT MERGERS}

We now investigate the effects of subsequent mergers following the first 1:1 mass merger A1, which are expected to drive the assembly of ellipticals (e.g. Naab et al. 2009; Naab \& Ostriker 2017). We extend the $N$-body integration of model A1 for a further $\sim 200$ Myrs to ensure that the surface luminosity profile is no longer evolving and core formation has halted. By this time, the separation between the SMBHs has become comparable to the softening length. We then estimate the coalescence time of the BHB due to the combined effects of three-body scatterings and emission of GWs. We adopt the semi-analytic model, referred to as the Continuous Coefficients Method (CCM), for the evolution of the binary's orbital elements presented in Nasim et al. (2020), which includes a time-dependent hardening rate. The $\mathrm{CCM}$ approximates the evolution of the $\mathrm{BHB}$ by using a time-dependent polynomial extrapolation to model threebody effects and the Post Newtonian prescription by Peters (1964) to model the effects of GW emission. The time-dependent polynomial extrapolation naturally accounts for different density profiles by fitting the hardening rate of the binary.

We find that the coalescence time for the BHB in model A1 is $\sim 3.5 \mathrm{Gyr}$, short enough that we can safely assume that a sequence of mergers can follow within a Hubble time. We then combined the two SMBHs into one, placed it at the binary's COM and modelled subsequent mergers on to the A1 remnant.

We first consider the B1-B4 mergers where the primary galaxy is the A1 remnant and the secondary is as described in Table 3. While $\mathrm{B} 1$ and $\mathrm{B} 2$ are 1:10 mergers, $\mathrm{B} 3$ is a 1:4 merger, and B4 a 1:2 merger. The secondary progenitor for $\mathrm{B} 1$ is set up using abundance matching, 

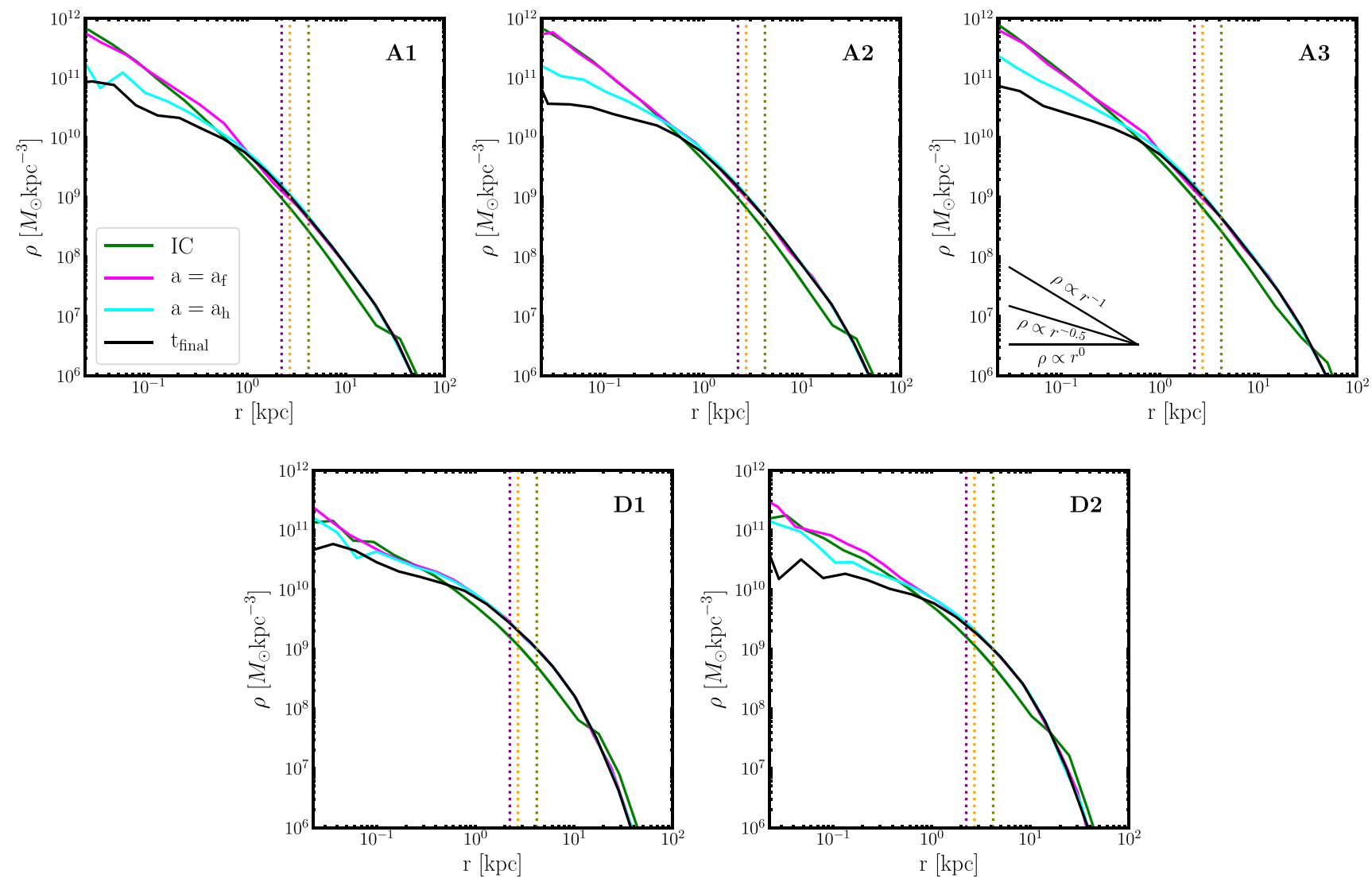

Figure 4. Spatial density profiles for the initial equal mass major mergers with SMBHs (A1-3, top panels) and the equal mass major mergers using D19 parameters: D1 and D2 (bottom left and bottom right panel, respectively). The vertical dotted lines are as defined in Fig. 3. Initial equal mass mergers with SMBHs form a weak central cusp in spatial density.

while the progenitors for remnants B2-B4 are scaled down versions of $\mathrm{A} 1 .^{6}$ We aim to determine whether cores grow over subsequent mergers, as generally expected for major mergers (Merritt 2006; De Lucia \& Blaizot 2007; Naab et al. 2009; Naab \& Ostriker 2017), or if they saturate instead, and what effects minor mergers have on the density profiles.

\subsection{Tidal deposition}

During a minor merger a sinking satellite has been shown to heat the primary galaxy, causing the density profile to become shallower or even forming a constant density core (Read et al. 2006). Because dynamical friction is suppressed in constant density cores, the satellite stalls at the edge of the core and never reaches the centre of the system (Antonini \& Merritt 2012; Dosopoulou \& Antonini 2017). Goerdt et al. (2010) also show that the core is formed at the radius where the enclosed stellar mass matches the mass of the satellite. Therefore, if a satellite is not dense enough to survive the infall to the centre, mass is deposited at the edge of the core, effectively enlarging its size. This represents a further mechanism of core formation, which we name tidal deposition, that does not operate by lowering the central density like binary scouring but rather by increasing the outer density. An additional key difference

${ }^{6} \mathrm{We}$ observe that mass segregation is not present in the numerical simulations with SMBHs, but was present in simulations without SMBHs. This motivated the A1E merger which contained equal mass particles without SMBHs. between the two mechanisms is the time at which they operate: while tidal deposition takes place during the merger, i.e. at early times in the evolution, binary scouring becomes efficient after the dynamical friction phase, when the SMBHs become bound.

We find evidence of tidal deposition in models B2-B4. The surface luminosity profiles of the B models are presented in the upper and middle left panels of Fig. 6. We observe no significant evolution in the central profiles of B1 and B2, however B2 shows evidence of deposition of mass by the time the separation $a_{\mathrm{f}}$ is reached. Model $\mathrm{B} 2$ hosts a more massive SMBH and stellar bulge than model B1, and this results in the faster infall seen in Fig. 2. The earlier tidal stripping experienced by B2 results in the observed modest deposition at large radii. This can also be seen in the Lagrangian radii (Fig. 5), where radius $r_{4}$ shows a small early decline while the innermost radii show no significant variations. However, the secondary galaxy is not sufficiently massive to affect the primary's profile in either model.

The more massive infallers in models B3-B4 result in a more noticeable tidal deposition at early times: the surface luminosity profiles show an increase in the outer regions by the time $a_{\mathrm{f}}$ is reached. There is also a small increase in the central density of the primary at early times which is due to the infall of the secondary galaxy. However, this is erased by binary scouring during the hardening phase, which results in a shallower final profile. Binary scouring plays a role in the 1:2 merger B4, as can be seen in the evolution of the $r_{1}$ Lagrangian radius in Fig. 5, but is negligible in model B3 due to the smaller SMBH mass. In order to verify this interpretation, we 

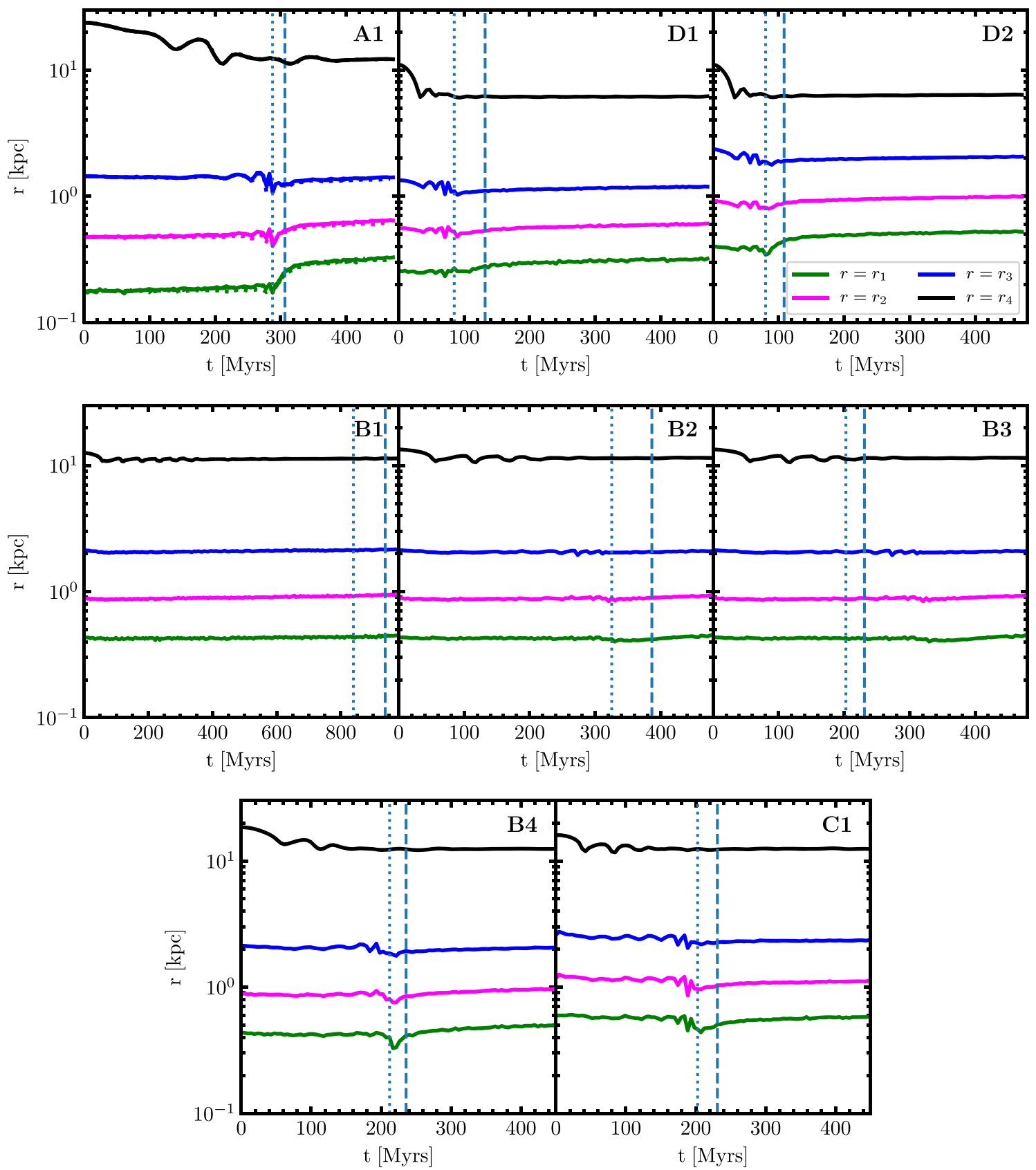

Figure 5. Evolution of the Lagrangian radii defined in equation (8) for the different merger remnants. The Lagrangian radii for merger runs $A 2$ and $A 3$ are represented by dotted/dashed lines in the upper left panel which approximately lie on top of the Lagrangian radii for model A1. The vertical dotted/dashed lines correspond to the times when $a=a_{\mathrm{f}}$ and $a=a_{\mathrm{h}}$ are reached, respectively. Both binary scouring and tidal deposition can be clearly seen in the Lagrangian radii. Scouring is particularly effective in the equal mass merger A1, whereas tidal deposition is significant in models D1 and D2, as well as in subsequent mergers. The time evolution also shows that tidal deposition operates at early times, while the merger is ongoing, while scouring requires an SMBH pair and operates after the end of the dynamical friction phase.

reran models B3 and B4 without an SMBH in the secondary galaxy (model B3a and B4a, see Table. 3). The surface luminosity profiles (presented in the central middle and right-hand panels of Fig. 6) show evidence of tidal deposition as well as a rise in the central density at early times, similarly to the models with a BHB. However, the central increase persists to late times as binary scouring does not operate in these models. The weak cusp observed in the spatial density profile from the initial major merger persists after the subsequent mergers, as shown in Fig. 7.
The tidal deposition we observe in the minor mergers following the first equal mass merger is in agreement with models of core formation in ellipticals galaxies via accretion at late times $(z<3)$, in which material is stripped from the secondary and is deposited on to the primary, thus increasing the size of the core. During this phase there is a reduction in the central concentration due to heating and dynamical friction from the initial surviving core (e.g. El-Zant, Shlosman \& Hoffman 2001; Naab et al. 2009; Naab \& Ostriker 2017). 

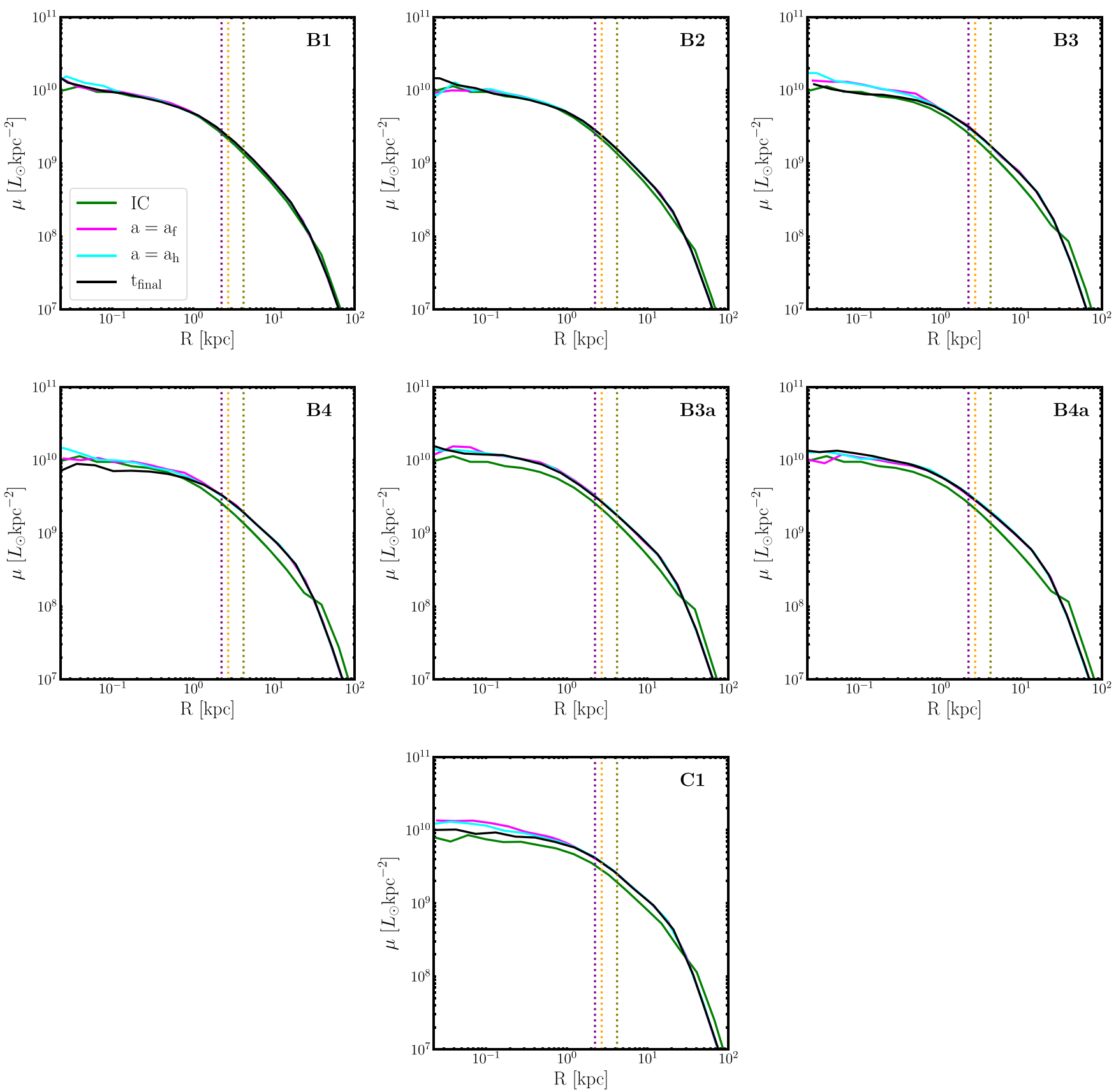

Figure 6. Surface luminosity profiles for the subsequent mergers: (B1-3, top panels), 1:2 major merger (B4, middle left panel), 1:4 and 1:2 mass mergers without secondary SMBH: B3a and B4a (central and middle right panel, respectively), 1:3 mass merger subsequent from B4 (C1, bottom panel). The profiles are computed at different times during the evolution: the initial condition (green line), the times when $a_{\mathrm{f}}$ (magenta) and $a_{\mathrm{h}}$ (cyan) are reached, and the end of the $\mathrm{N}$-body integration (black). The vertical dotted lines represent the three largest core radii known to date: IC 1101 (olive) with a $4.2 \mathrm{kpc}$ core, A2261-BCG (orange) with a $2.71 \mathrm{kpc}$ core, and 4C +74.13 (purple) with a $2.24 \mathrm{kpc}$ (Dullo 2019). Subsequent mergers with SMBHs primarily increase the size of the core via further tidal deposition. The mass deficit carved in the initial equal mass major merger does not grow linearly with the number of subsequent mergers. The subsequent mergers without BHBs increase their central concentration to absence of binary scouring. The surface mass densities can be computed by multiplying the surface luminosity by $\Upsilon=3.5 \mathrm{M}_{\odot} / \mathrm{L}_{\odot}$.

We note that tidal deposition is not observed in Rantala et al. (2019), who also investigated the effect of subsequent mergers. The reason for this is not fully understood, but likely owes to differences in the initial conditions.

Finally, we evolved the BHB formed in model B4 to coalescence following the same semi-analytic recipe adopted for model A1 and then proceeded to perform a subsequent 1:3 dry major merger $(\mathrm{C} 1)$ where the secondary progenitor is IC-1, with parameters as described in Table 3. The evolution of the surface luminosity profile of $\mathrm{C} 1$ is plotted in Fig. 6 (bottom panel). Perhaps surprisingly for a 1:3 major merger, we observe a qualitatively similar evolution to remnants B3 and B4, with evidence of tidal deposition at large radii and an increase in central density that persists to late times, with no binary scouring. This clearly indicates that scouring is not effective in a previously 

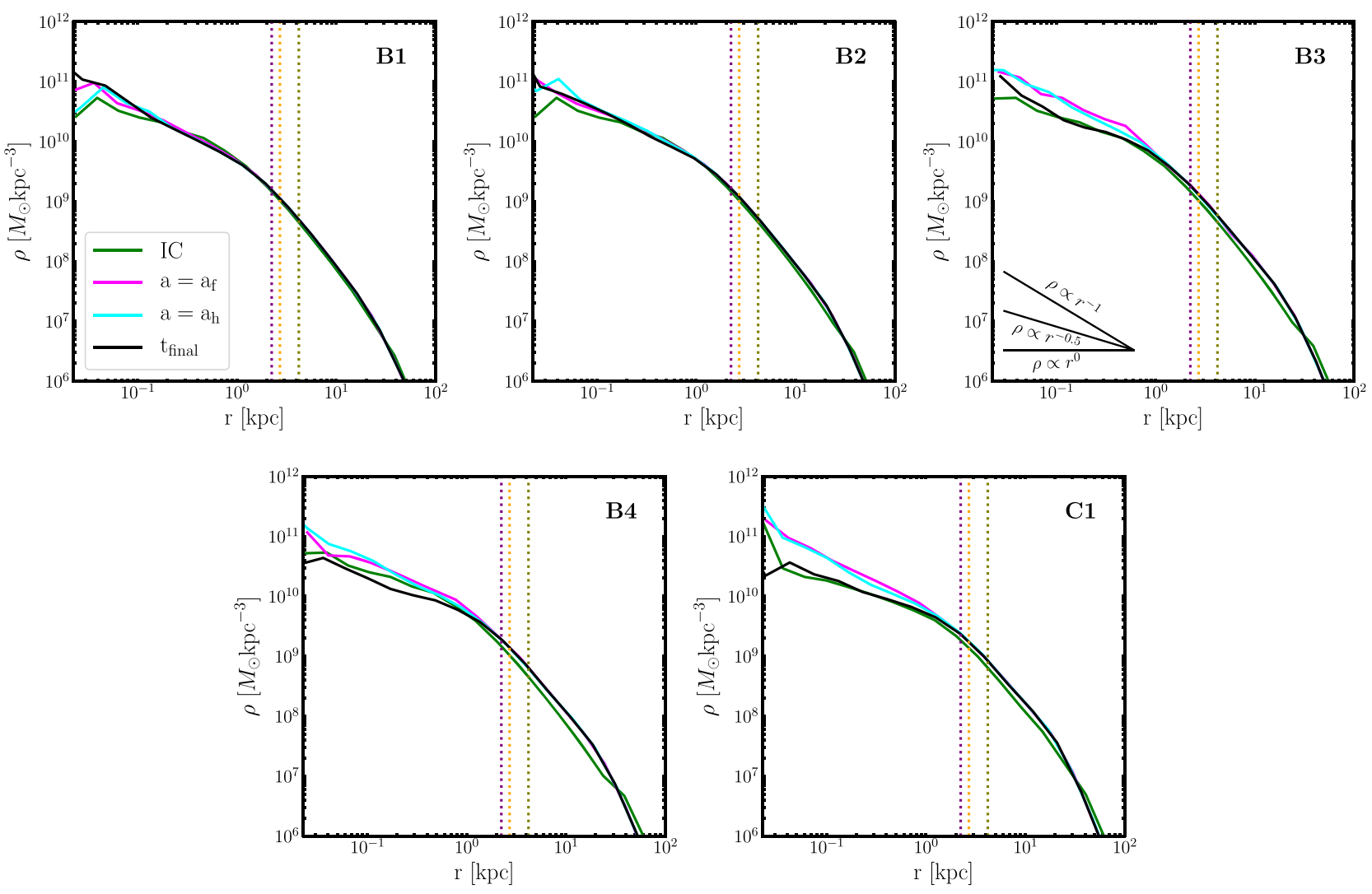

Figure 7. Spatial density profiles for the subsequent mergers: (B1-3, top panels), 1:2 major merger (B4, lower left panel), and 1:3 mass merger subsequent from B4 (C1, bottom right panel). The vertical dotted lines are as defined in Fig. 3. The spatial density profiles for the subsequent mergers show evidence for the formation of a weak central cusp which appears cored in projection.

carved core, and cores do not necessarily grow through subsequent mergers, as generally assumed in theoretical models (Merritt 2006; Rantala et al. 2019). For example, Merritt (2006) suggest that core size may be used as an indication of the number of major mergers experienced by a given galaxy. We find no evidence of this behaviour, and this is due to the fact that scouring is not effective in an already depleted core, carved by a previous merger. However, if cores saturate in flat profiles, independent mechanisms need to be invoked to explain large cores, with a mass deficit much larger than the assumed SMBH mass.

\subsection{Stalled satellites}

The photometric analysis of A2261-BCG shows a number of objects in the core region, referred to as 'knots' (Postman et al. 2012; Bonfini \& Graham 2016). Three of these objects (knots 1-3) lie along the line of sight within the core, causing a bump in the surface brightness profile (see fig. 1 of Bonfini \& Graham 2016). The authors suggest that, in the context of the stalled perturber scenario, the most massive knot, or all three combined, could be (at least partially) responsible for the large core observed in the galaxy as the mass contained within the core matches that of the knots. It was suggested in Dosopoulou \& Antonini (2017) and Burke-Spolaor et al. (2017) that these knots are stalled infalling satellites which have been stripped during their infall.

We plot the surface luminosity profiles in Fig. 8 at the times where these bumps are visible. While the bump in model B2 is quite small, due to the smaller mass of the secondary, we see prominent bumps for the larger mass ratio simulations (B3, B4, and C1). Interestingly, they are found at radii comparable to the largest known break radii for massive ellipticals. We find that these bumps persist, on average, for about 10 Myr (see Table 4), so they are generally short-lived compared to the duration of the merger. However, if a galaxy is observed while a smaller secondary is infalling, such bumps can be seen in the surface luminosity profile and can make the core appear larger. We then plot the stellar surface density of the subsequent mergers in Fig. 9 at the times where we observe the bumps in the luminosity profiles. The brighter regions represent the overdense infalling secondary while the centre of the primary galaxy remains approximately at the origin. These overdense structures are seen in all simulations featuring bumps in the surface luminosity profile, and resemble the observed knots in A2261-BCG.

We estimated the mass enclosed within these knot-like structures in order to compare them with the estimated observed mass determined in Bonfini \& Graham (2016). To do this, we centred on the tidally stripped secondary galaxy (Power et al. 2003) and estimated its radial extent by numerically calculating its tidal radius according to

$r_{t}=r_{\text {orbit }}\left(\frac{M_{\text {sat }}\left(<r_{t}\right)}{M_{\text {gal }}\left(<r_{\text {orbit }}\right)}\right)^{\frac{1}{3}}$.

We then adopt the stellar mass enclosed within the tidal radius as an estimate of the the spheroidal luminous mass of the knot structure, $M_{\text {knot }}$. These are given in Table 4 . We find that the mass of the secondary galaxy in remnant B2 is about half that of knot 3, whereas the secondary galaxies in the more massive remnants are 

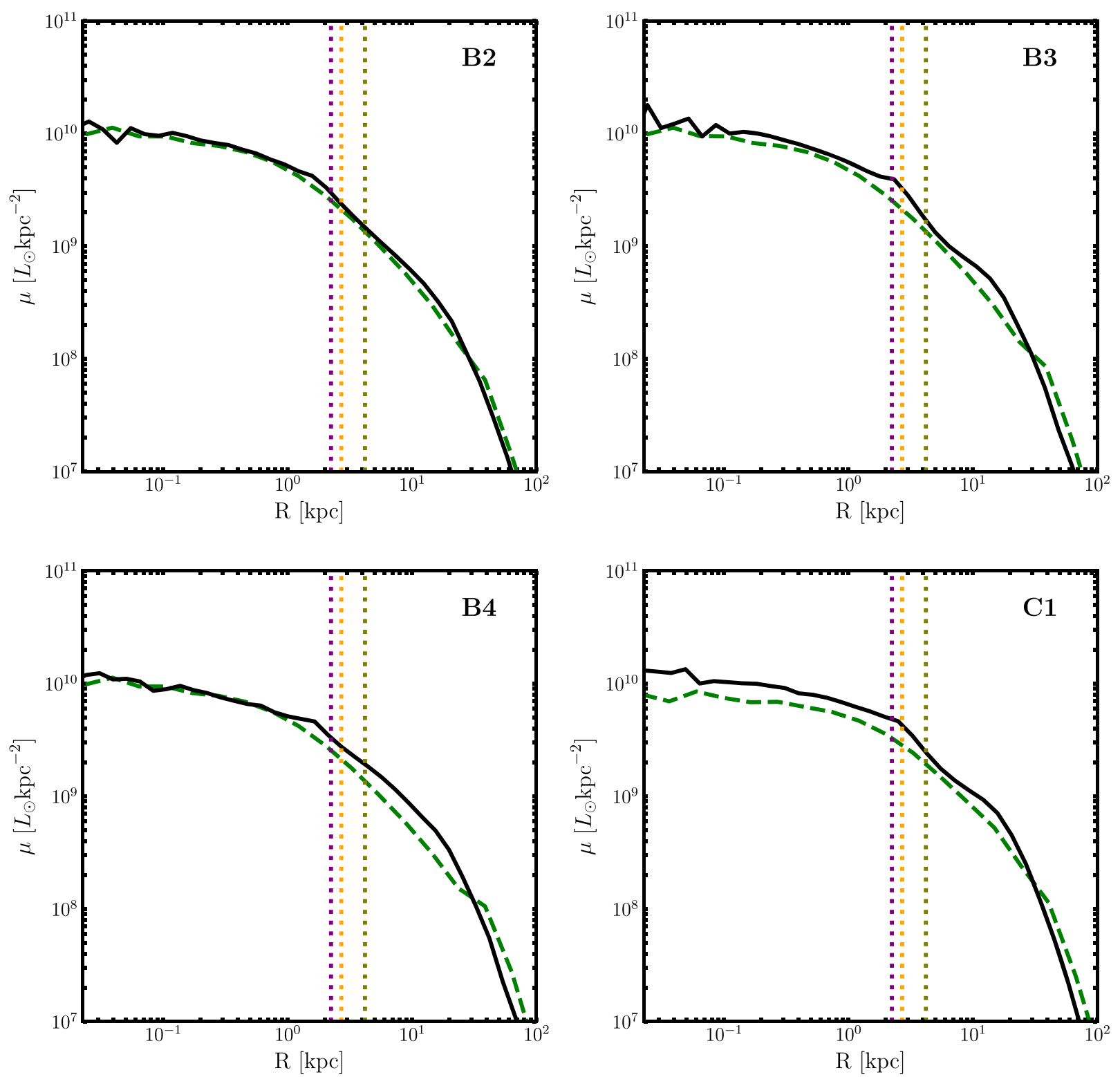

Figure 8. Surface luminosity profiles of subsequent mergers at times showing the formation of bumps (solid lines) to be compared against the initial profiles (dashed lines). As in Fig. 3, the vertical dotted lines represent the three largest core radii known to date: IC 1101 (olive) with a 4.2 kpc core, A2261-BCG (orange) with a $2.71 \mathrm{kpc}$ core, and $4 \mathrm{C}+74.13$ (purple) with a $2.24 \mathrm{kpc}$ (Dullo 2019). The observed bumps are due to the infall of satellites during minor mergers, and are observed in A2261-BCG (Postman et al. 2012; Bonfini \& Graham 2016). The surface mass densities can be computed by multiplying the surface luminosity by $\Upsilon=3.5 \mathrm{M}_{\odot} / \mathrm{L}_{\odot}$.

larger. It is therefore plausible that the knots observed by Bonfini \& Graham (2016) are tidally stripped satellites of independent accretion events. However, additional information like the age, metallicity, and relative velocity of the knots are required to better constrain their origin.

A significant implication of our results is that knot 3 should contain a central SMBH of mass $2.54 \times 10^{9} \mathrm{M}_{\odot} \leq M_{\bullet} \leq 6.35 \times 10^{9} \mathrm{M}_{\odot}$. It is unclear whether the smaller knots should contain central SMBHs, as they are significantly less massive. We also find that merger B1 (a 1:10 minor merger with parameters from abundance matching) does not show a 'bump' in the surface brightness profile due to the infalling satellite being less massive and not very dense. Low-mass satellites would need to be much denser than considered here to produce a visible bump in the profile.

\subsection{Core size}

Several different approaches can be found in the literature to identify and measure core sizes, both in simulations and observational data. A popular method to determine the core size is to fit the surface brightness profile of the galaxy with the Nuker-profile (Lauer et al. 1995)

$\mu(R)=\mu_{\mathrm{b}} 2^{(\beta-\gamma) / \alpha}\left(\frac{R}{R_{\mathrm{b}}}\right)^{-\gamma}\left[1+\left(\frac{R}{R_{\mathrm{b}}}\right)^{\alpha}\right]^{(\gamma-\beta) / \alpha}$,

where $R_{\mathrm{b}}$ is the break radius, $\mu_{\mathrm{b}}$ is the surface brightness at the break radius, $\gamma$ is the inner logarithmic slope of the profile, $\beta$ is the outer logarithmic slope, and $\alpha$ regulates the steepness of the transition between the outer and inner profile relative to the break radius. In 
Table 4. Break radii derived from the MCMC fitting procedure for the merger remnants described in Table 3. From left to right, the columns give the merger remnant simulation label; the core-Sérsic break radius at the time where $a=a_{\mathrm{f}}, R_{\mathrm{b} 1}$; the core-Sérsic break radius at the end

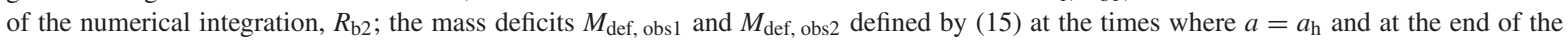
numerical integration; the mass deficits $M_{\mathrm{def}, \operatorname{sim} 1}$ and $M_{\mathrm{def}, \operatorname{sim} 2}$ defined by (14) and evaluated at the same times; the average lifetime of the bump formed in the surface luminosity profile due to the infall of the secondary galaxy, $t_{\text {bump }}$; the estimated mass of the knot according to the procedure outlined in 4.2, $M_{\mathrm{knot}}$; and the outer and inner slope parameters defined in equation (11) obtained from fitting the Core-Sérsic profile at the final $N$-body integration time, $n$ and $\gamma$. Mass deficits are given in units of the total BHB mass $M_{12}$.

\begin{tabular}{|c|c|c|c|c|c|c|c|c|c|c|}
\hline Merger remnant & $\begin{array}{c}R_{\mathrm{b} 1} \\
(\mathrm{kpc})\end{array}$ & $\begin{array}{c}R_{\mathrm{b} 2} \\
(\mathrm{kpc})\end{array}$ & $\begin{array}{c}M_{\text {def, obs } 1} \\
\quad\left(M_{12}\right)\end{array}$ & $\begin{array}{c}M_{\text {def, obs } 2} \\
\left(M_{12}\right)\end{array}$ & $\begin{array}{c}M_{\mathrm{def}, \operatorname{sim} 1} \\
\quad\left(M_{12}\right)\end{array}$ & $\begin{array}{c}M_{\mathrm{def}, \operatorname{sim} 2} \\
\quad\left(M_{12}\right)\end{array}$ & $\begin{array}{c}t_{\text {bump }} \\
\text { (Myrs) }\end{array}$ & $\begin{array}{c}M_{\text {knot }} \\
\left(10^{10} \mathrm{M}_{\odot}\right)\end{array}$ & $n$ & $\gamma$ \\
\hline A1 & 0.26 & 0.60 & 0.03 & 0.26 & 0.07 & 0.28 & - & - & 4.2 & 0.05 \\
\hline B1 & 0.52 & 0.60 & 0.20 & 0.22 & 0.03 & 0.04 & - & - & 4.1 & 0.19 \\
\hline B2 & 0.44 & 0.64 & 0.15 & 0.24 & 0.0006 & 0.05 & 4.7 & 2.4 & 3.8 & 0.16 \\
\hline B3 & 0.36 & 0.71 & 0.07 & 0.23 & -0.04 & -0.006 & 28.2 & 11.8 & 3.6 & 0.09 \\
\hline B4 & 0.35 & 0.87 & 0.06 & 0.31 & -0.018 & 0.10 & 23.5 & 13.8 & 3.6 & 0.06 \\
\hline $\mathrm{C} 1$ & 0.65 & 1.01 & 0.10 & 0.27 & -0.08 & -0.05 & 18.8 & 21.4 & 3.1 & 0.07 \\
\hline D1 & 0.33 & 0.61 & 0.02 & 0.09 & 0.017 & 0.10 & - & - & 2.5 & 0.03 \\
\hline D2 & 0.35 & 0.90 & 0.008 & 0.13 & 0.017 & 0.09 & - & - & 2.5 & 0.02 \\
\hline
\end{tabular}

this approach, once a core has been identified, the break radius $R_{\mathrm{b}}$ is used as a measure of the core size. However, Graham et al. (2003) suggest that the model parameters become unreliable when fit to light profiles with a large radial extent and the break radius shows a dependence on the radial fitting range.

An alternative approach is to fit the 6-parameter core-Sérsic profile (Graham et al. 2003; Trujillo et al. 2004)

$$
\mu(R)=\mu^{\prime}\left[1+\left(\frac{R_{\mathrm{b}}}{R}\right)^{\alpha}\right]^{\gamma / \alpha} \exp \left[-b\left(\frac{R^{\alpha}+R_{\mathrm{b}}^{\alpha}}{R_{\mathrm{e}}^{\alpha}}\right)^{1 /(\alpha n)}\right],
$$

with

$\mu^{\prime}=\mu_{\mathrm{b}} 2^{-\gamma / \alpha} \exp \left[b\left(2^{1 / \alpha} R_{\mathrm{b}} / R_{\mathrm{e}}\right)^{1 / n}\right]$,

where $\mu_{\mathrm{b}}$ is the intensity at the break radius $R_{\mathrm{b}}, R_{\mathrm{e}}$ is the effective radius, $n$ is the Sérsic index in the limit $R_{\mathrm{b}} \rightarrow 0$ (or $R \rightarrow \infty$ ), and $\alpha$ regulates the steepness of the transition between the outer slope $n$ and the inner slope $\gamma$ of the Sérsic profile. The identification of core galaxies, and the further measurement of core sizes, may differ in the two approaches. Break radii obtained by fitting the core-Sérsic model tend to be smaller (up to a factor 2-3) than the Nuker break radii (e.g. Trujillo et al. 2004; Dullo \& Graham 2012, 2013, 2014). As a result, 'cored' galaxies identified with the Nuker method may be classified as Sérsic spheroids with a smaller Sérsic index $n$ and no depleted core (Dullo \& Graham 2014). A non-parametric model (Carollo et al. 1997; Lauer et al. 2007) has also been proposed to estimate the core radius, referred to as the 'cusp' radius $r_{\gamma}$. This is defined as the radius where the logarithmic slope of the surface brightness profile is equal to $-1 / 2$ :

$\left.\frac{\mathrm{d} \log \mu(\mathrm{R})}{\mathrm{d} \log \mathrm{R}}\right|_{R=R_{\gamma}}=-\frac{1}{2}$

and is applied once the presence of a core has been established. It has been shown that this 'cusp' radius is consistent with the coreSérsic break radius $R_{\mathrm{b}}$ (Dullo \& Graham 2012; Rantala et al. 2018), though it can be smaller, especially in the case of very flat profiles (Gualandris \& Merritt 2012).

We choose to use the Core-Sérsic break radius as a measure of the core size for the surface luminosity profiles obtained from our simulated mergers. This allows us to make meaningful comparisons with observed cores. Following B16 and D19, we consider a radial fitting range $0.03 \mathrm{kpc}<\mathrm{R}<100 \mathrm{kpc}$.

We use a Markov Chain Monte Carlo (MCMC) approach to fit the core-Sérsic model to the surface luminosity data including uncertainties. Because the density profiles evolve with time, we measure the break radius at two different times: $R_{\mathrm{b} 1}$ at the time corresponding to the hard-binary separation and $R_{\mathrm{b} 2}$ at the end of the numerical integration. These are given in Table 4 . In the case of the major mergers, we find that larger cores are produced in D1 and D2 which use progenitor parameters from D19. This is expected based on their flatter surface luminosity profiles (see Fig. 3). The cores of the major mergers continue to grow after the BHB reaches the hard-binary separation due to stellar ejections, so that $R_{\mathrm{b} 2}>$ $R_{\mathrm{b} 1}$. The largest break radius, which is observed for model $\mathrm{D} 2$, is just under $1 \mathrm{kpc}$, much smaller than the $\sim 2-4 \mathrm{kpc}$ three largest observed cores.

The effect of the subsequent mergers depends sensitively on the mass ratio. For the smaller mass ratio mergers B1-B2, the break radii are largely unchanged from the preceding equal mass merger A1, as expected based on the similar surface luminosity profiles. This implies that the core size in these galaxies is set by the major merger and is preserved through subsequent 1:10 mergers. The core grows slightly in models B3 (1:3 merger) and B4 (1:2 merger), with a clear dependence on the mass ratio. Model $\mathrm{C} 1$, which is a further 1:3 major merger from remnant B4, shows only a small increase in core size. The core radii support our earlier conclusion based on the density profiles alone that cores do not necessarily grow through subsequent mergers. It appears that the size of the core is mainly set by the first equal mass merger, and further growth depends on the mass of the infalling satellite and the flatness of the surface density profiles. This is in apparent disagreement with the results of Rantala et al. (2019), who find that core formation is a cumulative effect over subsequent mergers.

The cores produced in our sequences of mergers are smaller than the largest $R_{\mathrm{b}} \geq 2 \mathrm{kpc}$ observed cores. While we see core growth over subsequent major mergers, we find that the number of major mergers required to form such large cores would be at odds with expectations from $\Lambda$ CDM (Naab et al. 2009; Naab \& Ostriker 2017). Note that we did not model subsequent 1:1 mergers as these would be unlikely from a cosmological point of view and would require unrealistically large galaxies as secondaries.

\subsection{The mass deficit}

The mass displaced by the BHB during the hardening phase is generally referred to as the 'mass deficit' and has been shown to be proportional to the combined mass of the binary (e.g. Milosavljević 

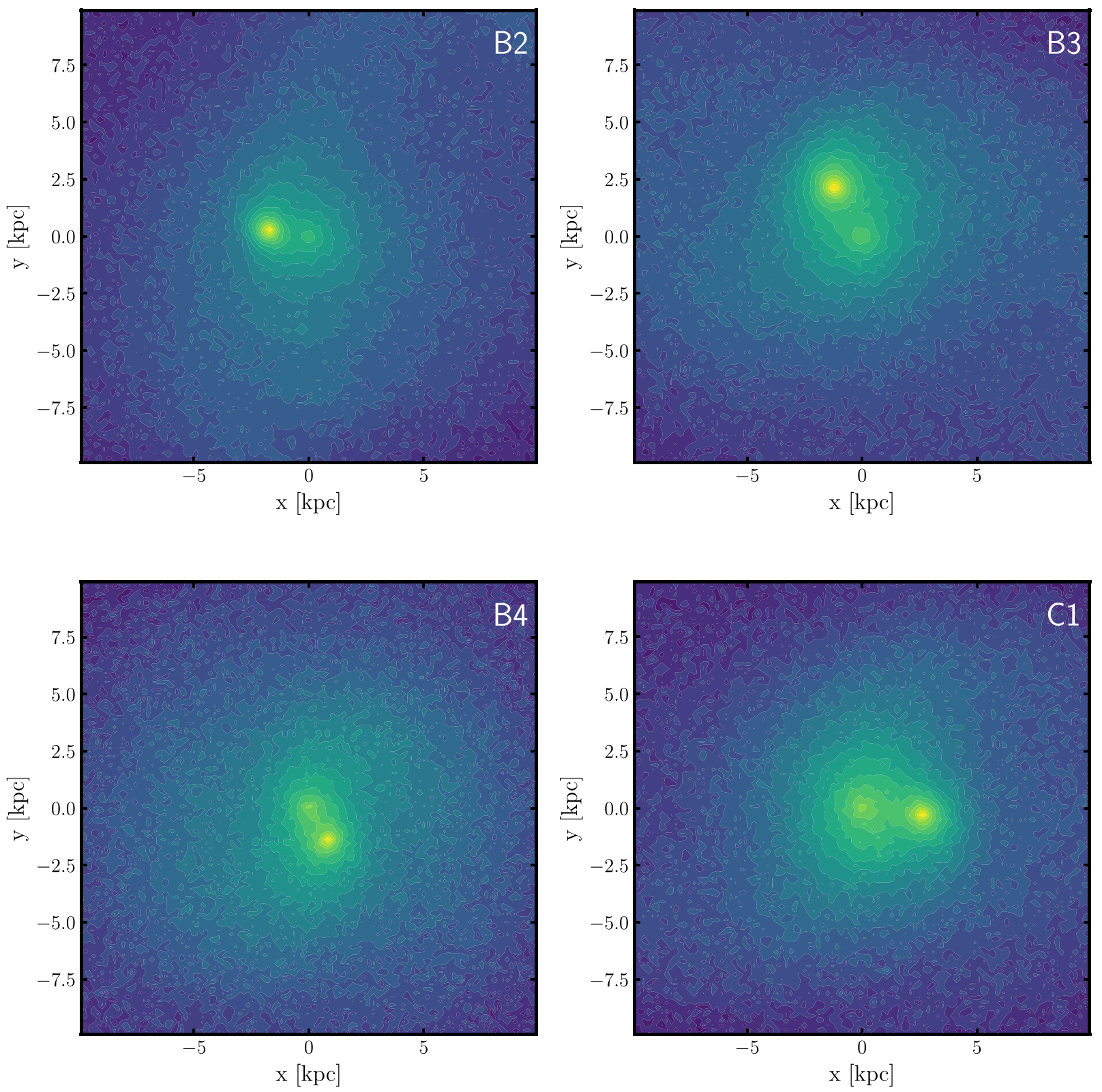

Figure 9. Stellar surface density of subsequent mergers at times where we observe bumps in the surface luminosity profiles (see Fig. 8). The infalling satellites are represented by the brighter overdense regions.

\& Merritt 2001; Graham et al. 2003; Merritt 2006). We adopt both a theoretical and observational approach to compute the mass deficit in the merger simulations. The former exploits the fact that we know the initial profile of the primary galaxy so we can compute the mass deficit as the difference in stellar mass enclosed within the core radius at a given time and in the initial profile. We then have that the mass deficit at time $t$ is

$$
M_{\text {def }}=\left.M_{\star}\left(r<r_{\text {core }}\right)\right|_{t=0}-\left.M_{\star}\left(r<r_{\text {core }}\right)\right|_{t}
$$

where $M_{\star}$ denotes the total mass in stars and $r_{\text {core }}$ the estimated core radius.

However, in order to allow a comparison with observational data we also adopt a surface-density-based approach in which first the luminosity deficit is computed as the difference between the integrated luminosity of the inward extrapolation of the Sérsic part of the core-Sérsic model compared to the luminosity of the coreSérsic model itself (e.g. Dullo \& Graham 2014; Bonfini \& Graham 2016; Dullo 2019). The mass deficit is then derived by applying an appropriate mass-to-light ratio $\Upsilon$

$M_{\mathrm{def}}=2 \pi \Upsilon \int_{0}^{R_{\mathrm{core}}}\left[\mu_{\mathrm{s}}(r)-\mu_{\mathrm{cs}}(r)\right] r \mathrm{~d} r$,

where $\mu_{\mathrm{s}}(r)$ and $\mu_{\mathrm{cs}}(r)$ are the surface luminosity profiles of the extrapolated Sérsic profile and the core-Sérsic profile (obtained from our MCMC fit), respectively, and we set $\Upsilon=3.5 \mathrm{M}_{\odot} / \mathrm{L}_{\odot}$ (Bonfini $\&$ Graham 2016). The normalization constant of the Sérsic profile is obtained by equating the Sérsic and core-Sérsic profiles at the break radius. 
We compute the mass deficit according to both approaches, which we call $M_{\text {def, sim }}$ (where 'sim' refers to simulations) and $M_{\text {def, obs }}$ (where 'obs' refers to observations) adopting the primary SMBH as centre and using the break radius obtained by our core-Sérsic fits as the core radius. We consider two separate times, the time when the hard-binary separation is reached and the end of the simulation. The results are listed in Table 4 as a fraction of the combined mass of the binary $M_{12}$.

The mass deficits computed with the two approaches are in good agreement for the initial 1:1 major mergers, though smaller than those found by Merritt (2006) in which $M_{\text {def }} \approx 0.5 M_{12}$. We attribute this discrepancy to a difference in initial conditions.

The subsequent mergers, however, reveal significant differences in the two computations, with the observed mass deficits being larger than the theoretical ones. This is due to the fact that the observational approach must rely on the assumption that the the primary galaxy followed a Sérsic profile prior to the merger, given by the inward extrapolation of the outer profile. Such assumption is clearly invalid in the case of subsequent mergers, where a core has already been carved by binary hardening in the previous major merger. The theoretical approach, on the other hand, adopts a realistic initial mass profile, as such information is readily available in the simulations. We see this effect clearly in the case of remnant B1 and A1, which show very similar surface density profiles (see Figs 3 and 6) but distinct estimates of the mass deficit.

Finally, note that the negative mass deficits listed in Table 4 are due to the increase in the central concentration of some models, namely B3, B4, and C1 (see Fig. 6).

\section{GRAVITATIONAL WAVE RECOIL}

Anisotropic emission of GWs results in a net kick velocity imparted to coalescing massive black holes (e.g. Bekenstein 1973). While the kick velocity is typically small $\left(\lesssim 200 \mathrm{~km} \mathrm{~s}^{-1}\right)$ for non-spinning BHs, it can reach $2000-5000 \mathrm{~km} \mathrm{~s}^{-1}$ for large spins with particular orientations (Campanelli et al. 2007; González et al. 2007; Lousto \& Zlochower 2011; Healy et al. 2018). This implies that massive black holes can recoil at a significant fraction of the galaxy's escape velocity, or even be ejected, dragging a small cluster of stars with them (Merritt et al. 2009). This stellar 'cloak' would most likely resemble a globular cluster or a sufficiently compact dwarf galaxy. The motion of a recoiling SMBH through the galaxy removes stars from the centre, turning a cuspy density profile into a core (e.g. Boylan-Kolchin et al. 2004; Merritt et al. 2004). If a core has already been carved by binary scouring in a previous major merger, the motion of the SMBH enlarges the core and mass deficits as large as five times the SMBH mass can be produced (Gualandris \& Merritt 2008). This is due to the fact that the $\mathrm{BH}$ transfers energy to the core at each passage, and its oscillatory motion dampens much less efficiently than expected from Chandrasekhar's theory (Chandrasekhar 1943) due to the flatness of the central profile (e.g. Read et al. 2006). Long-lived oscillations and enlarged cores are expected in most cases, since kicks exceeding the galaxy's escape velocity are very rare (Gerosa \& Sesana 2015).

While there is no confirmed detection of a recoiling $\mathrm{SMBH}$ yet, several candidates have been suggested over the years (e.g. Caldwell et al. 2014; Kalfountzou, Santos Lleo \& Trichas 2017; Chiaberge et al. 2018). Interestingly, the galaxies with the two largest cores, IC 1101 and A2261-BCG, show an offset between the outer isophotes and the cored region, consistent with simulations of repeated passages of an SMBH through the core (Dullo, Graham \& Knapen 2017; Dullo 2019). Postman et al. (2012) favour the ejected SMBH scenario for the formation of the core observed from A2261-BCG and suggest that one of the knots may be a stellar cluster surrounding an ejected SMBH.

In this section, we study the motion of recoiling SMBHs in two of our remnants, to investigate the effects of displaced black holes on the core size.

\subsection{Numerical simulations}

We consider merger remnant A1 and D2 and combine the two black holes into one as described in Section 4. We impart a recoil kick velocity to the newly formed $\mathrm{SMBH}$ following the prescription in Gualandris \& Merritt (2008), measured in units of the escape velocity $v_{\text {esc }}(r)=\sqrt{-2 \Phi(r)}$, where $\Phi(r)$ is the total gravitational potential due to both the stellar and dark matter particles. The kick is given, arbitrarily, in the $x$-direction, and a correction is made for the momentum introduced into the system. The magnitude of the kick is parametrized in units of the escape velocity from the centre of the galaxy, as detailed in Table 5, as we make no assumption on the magnitude and orientation of the spins prior to coalescence. The escape velocities of the SMBHs from the centre of remnant A1 and $\mathrm{D} 2$ are $v_{\mathrm{esc}} \approx 3840 \mathrm{~km} \mathrm{~s}^{-1}$ and $v_{\mathrm{esc}} \approx 4200 \mathrm{~km} \mathrm{~s}^{-1}$, respectively. No spin is given to the $\mathrm{SMBH}$ given that spin effects are only important in the immediate vicinity of the hole, a region we do not resolve. We follow the evolution of the kicked black holes with GRIFFIN until either the SMBH settles at the COM of the system or the integration time exceeds 2 Gyr. The parameters of the simulations are listed in Table 5 .

\subsection{Core size and mass deficit}

Due to the combined effects of the kick and dynamical friction, the SMBHs exhibit damped oscillatory motion, in agreement with the results of Gualandris \& Merritt (2008). For kicks larger than about $0.3 v_{\text {esc }}$, the black holes reach beyond the core and experience multiple oscillations that displace stellar mass, enlarging the preexisting core at each passage. The SMBH carries any mass bound to it in its excursions out of the core.

The surface luminosity profiles of the GW recoil models are shown in Fig. 10, computed relative to the COM of the system (all stars and DM particles). ${ }^{7}$ In order to study the evolution of the core region over time, we show the surface luminosity profiles at four distinct times: the initial profile (green); the time of maximum displacement of the SMBH from the COM (magenta); the time of the first return to the centre (cyan); and the end of the $N$-body integration (black). All models show a flattening of the inner profile and the formation of a core, roughly proportional to the magnitude of the kick. The first excursion of the SMBH to apocentre has the largest effect on the central density, followed by smaller changes with subsequent passages through the core. We observe long-lived oscillations for all cases of large kicks $\left(v_{\text {kick }}=0.6-0.9 v_{\text {esc }}\right)$, in agreement with Gualandris \& Merritt (2008), resulting in a slow but prolonged increase in the core size.

We fit core-Sérsic profiles to the surface luminosity profiles of the B-models (obtained from remnant A1) and the D-models (obtained

\footnotetext{
${ }^{7} \mathrm{We}$ also considered the profiles centred on the SMBH and the centre of density (COD) of the stellar component. We find a very good agreement between the profiles centred on the COM and COD at all times, and also a good agreement for all three centering choice when the SMBH is at its pericentre passage within the core. We find that when the SMBH is exterior to the core region, centring on the SMBH yields non-physical profiles.
} 
Table 5. Parameters of the GW recoil simulations. The B-GW and D-GW models are produced from the merged remnants $\mathrm{A} 1$ and $\mathrm{D} 2$, respectively. The kick velocity $\mathrm{v}_{\mathrm{kick}}$ is given in units of the escape velocity $v_{\mathrm{esc}}$ from the centre. The break radii $R_{\mathrm{b}, \mathrm{GW} 1}$ and $R_{\mathrm{b}, \mathrm{GW} 2}$ are evaluated at the time when the $\mathrm{SMBH}$ is at the first apocentre/pericentre passage with respect to the COM of the system, while $R_{\mathrm{b}, \mathrm{GW} 3}$ is evaluated at the end of the numerical integration. The mass deficits $M_{\mathrm{def}, \text { obs } 3}$ and $M_{\mathrm{def}, \text { sim3 }}$ are calculated using equation (15) and equation (14), respectively, both at the end of the numerical integration. $R_{\mathrm{b}, \text { ini }}$ is the initial break radius of the remnant prior to SMBH ejection. The escape velocity from the centre of A1 and D2 are $v_{\mathrm{esc}} \approx 3840 \mathrm{~km} \mathrm{~s}^{-1}$ and $v_{\mathrm{esc}} \approx 4200 \mathrm{~km} \mathrm{~s}^{-1}$, respectively.

\begin{tabular}{lcccccccc}
\hline Run & $\begin{array}{c}\mathrm{v}_{\text {kick }} \\
\left(\mathrm{v}_{\mathrm{esc}}\right)\end{array}$ & $\begin{array}{c}R_{\mathrm{b}, \mathrm{GW} 1} \\
(\mathrm{kpc})\end{array}$ & $\begin{array}{c}R_{\mathrm{b}, \mathrm{GW} 2} \\
(\mathrm{kpc})\end{array}$ & $\begin{array}{c}R_{\mathrm{b}, \mathrm{GW} 3} \\
(\mathrm{kpc})\end{array}$ & $\begin{array}{c}M_{\mathrm{def}, \mathrm{obs} 3} \\
\left(M_{\bullet}\right)\end{array}$ & $\begin{array}{c}M_{\mathrm{def}, \text { sim3 }} \\
\left(M_{\bullet}\right)\end{array}$ & $\begin{array}{c}R_{\mathrm{b}, \text { ini }} \\
(\mathrm{kpc})\end{array}$ & Merger remnant \\
\hline B-GW01 & 0.1 & 0.81 & 0.82 & 0.82 & 0.50 & 0.38 & 0.60 & $\mathrm{~A} 1$ \\
B-GW03 & 0.3 & 1.23 & 1.27 & 1.32 & 0.94 & 1.26 & 0.60 & $\mathrm{~A} 1$ \\
B-GW06 & 0.6 & 1.27 & 1.35 & 1.42 & 1.00 & 1.51 & 0.60 & $\mathrm{~A} 1$ \\
B-GW07 & 0.7 & 1.27 & 1.37 & 1.44 & 1.02 & 1.55 & 0.60 & $\mathrm{~A} 1$ \\
B-GW08 & 0.8 & 1.29 & 1.37 & 1.48 & 1.05 & 1.58 & 0.60 & $\mathrm{~A} 1$ \\
B-GW09 & 0.9 & 1.32 & 1.40 & 1.53 & 1.08 & 1.60 & 0.60 & $\mathrm{~A} 1$ \\
D-GW03 & 0.3 & 2.21 & 2.42 & 2.64 & 2.70 & 0.86 & 0.90 & D2 \\
D-GW08 & 0.8 & 2.67 & 2.78 & 2.96 & 3.30 & 1.38 & 0.90 & D2 \\
\hline
\end{tabular}

from remnant D2) to obtain the break radii given in Table 5 . Similarly to the surface luminosity profiles, these are evaluated at three significant times in the evolution: the time of the first apocentre passage of the SMBH, the time of the first return to the centre, and the end of the integration. We again see evidence that most of the core is carved during the first excursion, for kicks large enough to drive the SMBH out of the core with slowly damped oscillations. For the B-GW models with kick velocities large enough to drive the $\mathrm{SMBH}$ out of the core, we find an increase in the final break radius of a factor 2-2.5 with respect to the initial one, depending on the magnitude of the kick. While this represents a significant effect, it is not sufficient to explain the core size measured in A2261-BCG. For the D-GW models, on the other hand, we find break radii as large as $3 \mathrm{kpc}$, making this mechanism a plausible explanation for the largest cores known to date.

The mass initially bound to the black hole, i.e. just after the kick is imparted, is shown in Fig. 11 for all models considered in Table 5. In agreement with previous studies (Boylan-Kolchin et al. 2004; Merritt et al. 2004; Gualandris \& Merritt 2008; Merritt et al. 2009), it decreases steeply with increasing $\mathrm{v}_{\text {kick}}$. The models derived from the D2 remnant are characterized by a larger bound mass, as expected from the larger SMBH mass. However, the bound mass for such models is also larger when given in units of the SMBH mass, as shown in the figure, implying that a larger core and flatter profile also result in a larger bound mass.

Interestingly, we find that most of the central surface luminosity depletion occurs by the time of the first apocentre passage the SMBH makes with respect to the cored region, where subsequent pericentre passages only cause smaller additional depletions. This behaviour is not in agreement with the results of Gualandris \& Merritt (2008), who found that the primary mechanism of increasing the core size is the subsequent passages the SMBH makes through the core. We attribute this difference to the very different initial conditions adopted in this study, where we have multicomponent models with the inclusion of a dark matter halo as well as a very large core already in place prior to the GW recoil. More mass can be bound to the SMBH in a cored profile due to the larger tidal radius, and dynamical friction is suppressed in flat density profiles. This is clearly observed in the D-GW models which start out with a flatter profile compared to the B-GW models and show a reduced effect of subsequent SMBH passages through the cores. We also find that the bound stellar mass fraction, measured when the SMBH is at apocentre, increases slowly over time, due to increasing captures as the SMBH slows down.
The final core size is determined by the velocity of the kick, the SMBH mass and the slope of the central profile at the time of the recoil. We fit a power-law relation of the type

$$
\frac{R_{\mathrm{b}}}{R_{\mathrm{b}, \text { in }}}=K\left(\frac{\mathrm{v}_{\text {kick }}}{\mathrm{v}_{\mathrm{esc}}}\right)^{\alpha}+1 \text {, }
$$

valid for $0 \leq \mathrm{v}_{\text {kick }} \leq \mathrm{v}_{\mathrm{esc}}$, where $R_{\mathrm{b} \text {, in }}$ is the break radius prior to the kick and $K$ is a constant which incorporates the dependence on both the SMBH mass and the central slope. We find best-fitting values of $\alpha=0.42, K=2.02$ in the B-GW models and $\alpha=0.18, K=2.41$ in the D-GW models, due to the larger SMBH mass and flatter profiles in the D2 remnant.

We proceed to calculate the mass deficits at the end of the numerical integration according to equations (14) and (15). We find that these increase with increasing kick velocity (see Table 5), in agreement with previous studies (Merritt et al. 2004; Gualandris \& Merritt 2008; Merritt et al. 2009). For the B-GW models and for any kick larger than $0.3 v_{\text {esc }}$, we find that the mass deficits derived from the core-Sérsic fits are systematically smaller than the mass deficits derived from the enclosed mass. The opposite is seen in the D-GW models, where the observational approach gives mass deficits more than twice as large as the enclosed mass approach. While this is a natural consequence of the large preexisting core present in these models, it implies that mass deficits estimated according to equation (15) are not a reliable measure of the mass displaced in the last merger experienced by the galaxy. Computing the mass deficit with respect to the inward extrapolation of the outer Sersic profiles simply provides an estimate of the mass displaced with respect to such profile, which is not a realistic assumption in the case of pre-existing cores and/or multiple mergers. We argue therefore that mass deficits computed in this way should be treated with caution when attempting to reconstruct the merger history of a galaxy, and that break radii represent a more robust measurement.

We note that the cores produced by GW recoil are different, in nature, from those produced by galaxy mergers. They correspond to genuinely flat profiles in spatial density, and this is confirmed by an Abel de-projection analysis of the surface density profiles.

\section{DISCUSSION}

Massive galaxies have long been theorized to form via hierarchical mergers of smaller systems (e.g. Toomre 1977; White \& Rees 1978). 

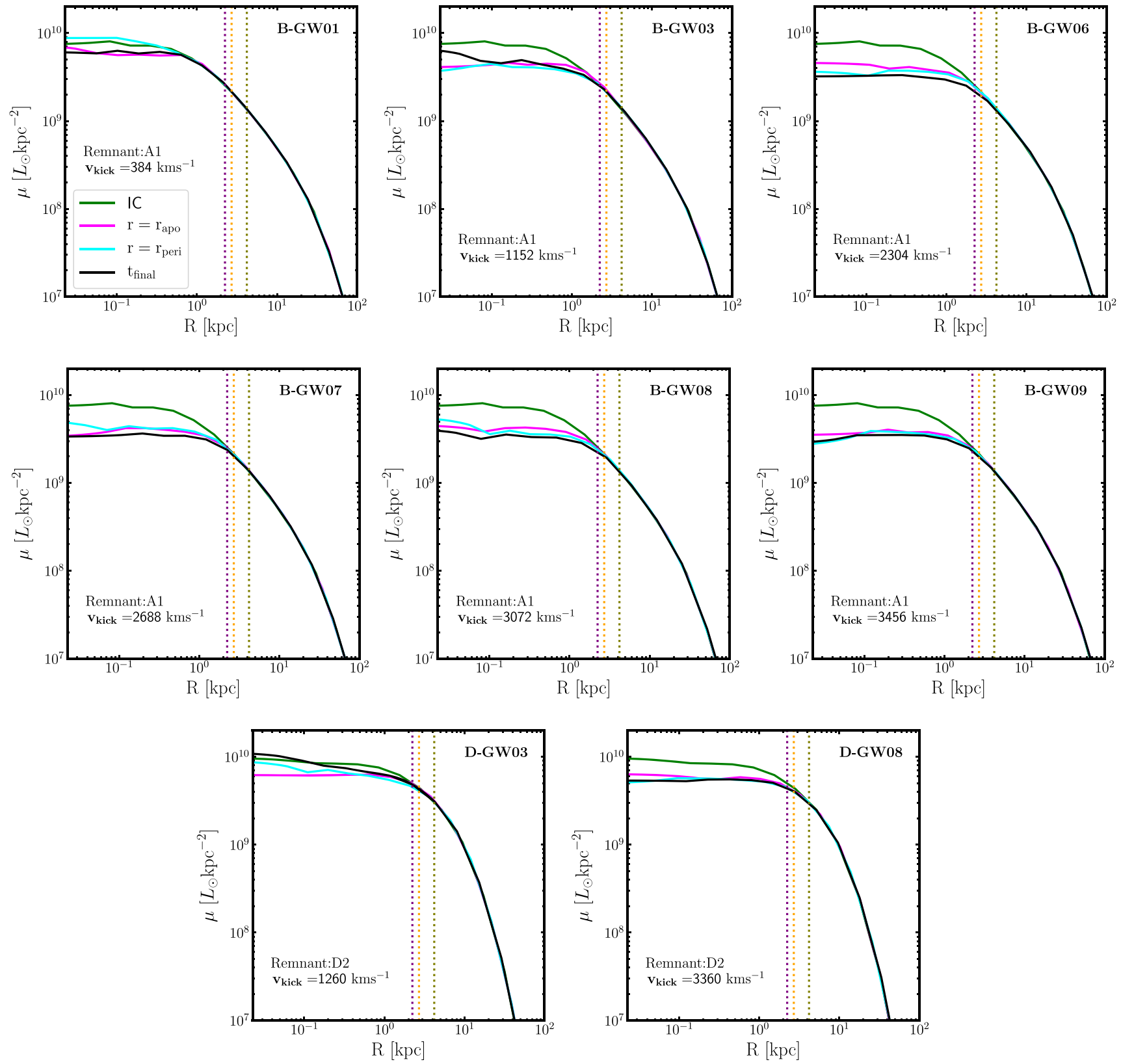

Figure 10. The surface luminosity profiles for the GW recoil simulations, with models as described in Table 5. The profiles refer to different times during the evolution: initial profile (green), the time of the maximum displacement of the SMBH from the COM of the system (magenta), the time of the first return of the SMBH to the core (estimated as the time of the first pericentre with respect to the COM) (cyan), and the final time of the $N$-body integration (black). The vertical dotted lines are as defined in Fig. 3. GW recoil of the SMBH causes a significant central depletion in the surface brightness profiles and enlarges the pre-existing core. In the case of the models derived using the D19 parameters, the core size obtained is comparable to the largest depleted cores known to date. The surface mass densities can be obtained by multiplying the surface luminosity by $\Upsilon=3.5 \mathrm{M}_{\odot} / \mathrm{L}_{\odot}$.

BCGs, which are found at the centre of massive galaxy clusters, are expected to have experienced significant accretion and merger events with smaller low-luminosity galaxies (Von Der Linden et al. 2007; Oliva-Altamirano et al. 2015). This implies that a significant fraction of large cored galaxies are BCGs, as recently confirmed by Dullo (2019) who found that $\sim 77$ per cent of large cored galaxies in their sample are BCGs. While cores larger than $0.5 \mathrm{kpc}$ are rare, A2261BCG stands out for its $2.7-3.6 \mathrm{kpc}$ core (Bonfini \& Graham 2016; Dullo 2019).
We have explored three scenarios of core formation in massive galaxies, with the goal of explaining the very largest observed cores: binary scouring, tidal deposition and GW-induced recoil. All progenitor galaxies in this study were modelled as multicomponent systems including a central SMBH, a spheroidal bulge following a Sérsic profile, and a dark matter halo described by the NFW profile, with parameters selected to mimic those of A2261-BCG. All of the mergers, including both major and minor mergers, and recoil simulations were performed self-consistently using the GRIFFIN code. 


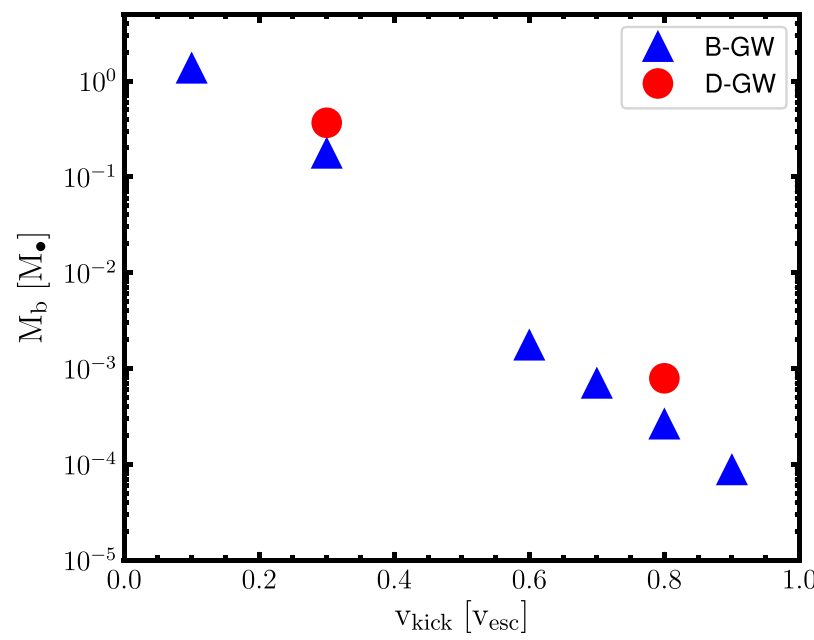

Figure 11. Initial stellar mass bound to the recoiling SMBH for the B-GW models (blue triangles) and D-GW models (red circles).

\subsection{Cores from binary scouring}

We find that binary scouring is effective in the first major merger in ejecting stellar mass from the central region, thereby lowering the central density and producing what, in projection, appears like a core. This process is responsible for turning a cuspy profile into a core, with typical sizes up to $\sim 0.9 \mathrm{kpc}$. While binary scouring can operate in subsequent mergers, we find that its effectiveness is greatly reduced once a core is already present in the primary galaxy. Cores may then grow in subsequent mergers due to tidal deposition by massive infalling satellites.

The observed size of the core cannot, therefore, be used to estimate the number of major mergers experienced by a galaxy, as assumed in works such as Merritt (2006). This also implies that binary scouring alone is not sufficient to explain the largest observed cores, and other mechanisms need to be invoked.

\subsection{Cores from tidal deposition and the origin of bright central 'knots'}

Minor mergers, which are more abundant in typical merger trees, produce bumps in the surface luminosity profiles and can somewhat increase the size of the core due to tidal deposition. Indeed, infalling satellites represent a plausible explanation for the peculiar 'knots' observed in A2261-BCG (Postman et al. 2012; Bonfini \& Graham 2016; Dosopoulou \& Antonini 2017). Bumps typically persist for a few tens of Myr in our simulations, but this will vary with the mass of the satellite.

According to our simulations, the most massive knot structure (knot 3 ) could contain a central SMBH with an estimated mass $2.54 \times$ $10^{9} \mathrm{M}_{\odot} \leq M_{\bullet} \leq 6.35 \times 10^{9} \mathrm{M}_{\odot}$. Smaller knots may not contain an SMBH due to their lower mass. We find that lower mass satellites do not produce observable bumps in our simulations, and we expect that they would need to be much denser to have an observable effect.

\subsection{Cores from gravitational wave recoil and an alternative model for bright 'knots'}

Recoil due to anisotropic GW emission significantly enlarges the core scoured during the phase of binary hardening. The core size scales with the kick velocity to the power $\sim 0.15-0.3$, but is also a function of the black hole mass and the slope of the central density profile, with larger cores produced in flatter profiles. Our galaxy models, adopting the structural parameters and SMBH mass by Dullo (2019), naturally produce cores as large as $\sim 3 \mathrm{kpc}$ for the largest kick velocities. We are therefore able to explain the largest observed cores if a major merger leading to binary scouring and coalescence of the holes is followed by GW recoil of the newly formed SMBH, with a kick velocity in excess of $\sim 1000 \mathrm{~km} \mathrm{~s}^{-1}$. Such large kick velocities are likely very rare as they arise only from peculiar orientations of the spins of the merging black holes (González et al. 2007; Healy et al. 2018; Lousto \& Healy 2019). However, only four galaxies are known with core radii larger than $2 \mathrm{kpc}$, implying that extremely large cores are also rare.

Interestingly, the galaxies with the three largest cores known to date show an offset in their outer isophotes with respect to the cored region (Postman et al. 2012; Dullo 2019; Gultekin et al. 2021). An offset is naturally expected as a result of the oscillatory motion of a recoiling $\mathrm{SMBH}$.

The knots observed in A2261-BCG could be due to the ejection of SMBHs carrying along an envelope of bound stars, called 'HyperCompact Stellar Systems' (HCSS) (Komossa 2012). These HCSSs have sizes and luminosities similar to globular clusters (or ultra compact dwarf galaxies in extreme cases of near escape velocity kicks) but have larger velocity dispersions (Merritt et al. 2009). They can be distinguished from tidally stripped galactic nuclei because of their compact nature and large velocity dispersions. Interestingly, the GW kick velocity can be estimated from the observed broadening of the absorption line spectrum of an HCSS due to internal motion (Merritt et al. 2009). We note that a compact cluster of stars surrounding the SMBH is not present in our initial conditions, but is a result of the oscillatory motion of the black hole through the galaxy. The predicted luminosities of HCSSs are however much lower than those of the knots in A2261-BGC (Merritt et al. 2009). The luminosity of the stellar cluster bound to the SMBH in the DGW08 model is $\sim 1.8 \times 10^{8} \mathrm{~L}_{\odot}$, a few times larger than the most luminous cases in (Merritt et al. 2009). Therefore, we consider this interpretation of the origin of the observed knots unlikely. BurkeSpolaor et al. (2017) took HST spectra of three of the four knots to test whether a recoiling SMBH is a viable explanation. The kinematics, colours, and masses of knots 2 and 3 are consistent with infalling low-mass galaxies or tidally stripped larger galaxies. An HCSS origin cannot be ruled out for knot 1 due to large errors in the measurement of the velocity dispersion. VLA radio imaging shows a compact off-centre radio source that appears to be the relic of an old AGN.

Our largest core is somewhat smaller than that derived by Bonfini \& Graham (2016). However, the authors adopt a single-component galaxy model for their fits, biasing their break and half-light radii towards larger values. A smaller break radius is obtained by fitting the galaxy with a multicomponent core-Sérsic spheroid and an exponential outer halo profile (Dullo 2019).

\subsection{The trouble with observed 'mass deficits' and 'cored' galaxies}

The mass deficit in massive elliptical galaxies has been used as a measure of the core size, in addition or instead of the break radius obtained from fitting a core-Sérsic profile. While this can be computed trivially in $\mathrm{N}$-body simulations as the difference in mass between the initial and final density profile, the observational approach must make an assumption about the initial profile. This is usually taken to be the integrated mass between inward extrapolation of the outer Sérsic profile assuming a mass-to-luminosity ratio (e.g. Dullo \& Graham 2013, 2014; Bonfini \& Graham 2016). While this is a reasonable 
assumption in major mergers where a core is first formed through binary scouring from a cuspy profile, it necessarily fails in subsequent mergers, both minor and major, where little mass is displaced from the central region. We therefore argue that the mass deficit cannot be directly used to constrain the merger history of a galaxy.

We emphasize once more that the presence of a flat core in the surface density profile of a galaxy, which is then traditionally defined a core galaxy (Ferrarese et al. 1994; Lauer et al. 1995), does not imply a constant density region in the central space density. In fact, any spatial density as steep as $\rho(r) \sim r^{-1}$ projects on to a seemingly flat profile in surface density (Milosavljević et al. 2002). We find that our models are well represented by a central power-law profile of the type $\rho(r) \sim r^{-0.5}$, in full agreement with both theoretical models (Merritt \& Fridman 1995) and observations (e.g. Lauer et al. 1995).

\subsection{The need for overmassive black holes}

The sequence of mergers that resulted in the largest cores in our simulations adopted an overmassive SMBH that lies above the $M_{\bullet}-\sigma$ and $M_{\bullet}-$ L relations for $M_{\bullet} \geq 10^{10} \mathrm{M}_{\odot}$ (Thomas et al. 2016; Dullo et al. 2017; Rantala et al. 2018; Dullo 2019). Recently the most massive SMBH was observed in Holm 15A with $M_{\bullet}=(4.0 \pm 0.8) \times$ $10^{10} \mathrm{M}_{\odot}$ which is approximately nine times larger than expected from the $M_{\bullet}-\sigma$ relation and four times larger than expected from the stellar mass relation (Mehrgan et al. 2019). Overmassive black holes are not fully understood but may arise from compact 'blue nugget' galaxies at high redshift $(z \geq 6)$, where high velocity dispersions allow SMBHs to reach larger masses (King \& Nealon 2019). Slowspinning SMBHs are expected to be the most massive, as these are less efficient at producing feedback in the form of outflows (Zubovas \& King 2019). The need for overmassive black holes in producing the largest observed cores is motivated by the approximately linear dependence of core sizes carved by both binary scouring (Merritt 2006) and GW recoil on SMBH mass (Gualandris \& Merritt 2008). In principle a different sequence of mergers and/or a larger GW recoil velocity might have produced a larger core than the one obtained here. However, we do not consider these effects sufficient to produce the largest cores without an overmassive SMBH.

\subsection{Cores from SMBH triples}

An additional mechanism able to produce large cores is scouring from SMBH triples (e.g. Hoffman \& Loeb 2007; Kulkarni \& Loeb 2012). These form whenever a merger with a third galaxy occurs before the coalescence of a BHB. The dynamical evolution of such triples depends sensitively on the masses and configuration of the system. If the orbit of the intruder is highly inclined with respect to that of the inner binary, Kozai-Lidov oscillations lead to high eccentricity at pericentre and trigger coalescence of the SMBHs before a close three-body encounter can take place (Iwasawa, Funato \& Makino 2006; Hoffman \& Loeb 2007). In most other cases, a strong and often resonant encounter takes place, with significant energy transfer leading to hardening of the inner binary and ejection of one SMBH. The lightest SMBH usually escapes while the binary recoils. Escape of all three SMBHs is extremely rare (Hoffman \& Loeb 2007). $N$-body simulations of SMBH triples in gas-free major mergers show enhanced core scouring with larger mass deficits than binary scouring and up to $\sim 5 M_{\text {• }}$ (Iwasawa, Funato \& Makino 2008). Kulkarni \& Loeb (2012) find that triple or multiple SMBHs are more likely at high redshift and in more massive galaxies. Using $\mathrm{N}$ body simulations with cosmologically motivated initial conditions, they show that haloes with present day mass of $10^{14}\left(10^{15}\right) \mathrm{M}_{\odot}$ have a
40 per cent (50 per cent) probability of having more than two SMBHs at redshift $2<z<6$. Triple SMBH scouring is therefore a possible, though rare, mechanism for producing large cores in massive galaxies at high redshift.

\section{CONCLUSIONS}

We have explored three scenarios of central core formation in massive galaxies, with the goal of explaining the very largest observed cores: binary scouring, tidal deposition, and GW-induced recoil. All progenitor galaxies in this study were modelled as multicomponent systems including a central SMBH, a spheroidal bulge following a Sérsic profile, and a dark matter halo described by the NFW profile, with parameters selected to mimic those of A2261-BCG, a giant elliptical galaxy with a central surface brightness core of size $\sim 3 \mathrm{kpc}$. All simulations were performed self-consistently using the GRIFFIN code.

We find that we can only produce the large surface brightness core of A2261-BCG, as measured by Dullo (2019), with the combination of a major merger that produces a $\sim 1 \mathrm{kpc}$ core through binary scouring, followed by the subsequent GW recoil of the SMBH that acts to then grow the core size to $\sim 3 \mathrm{kpc}$ as the SMBH oscillates about the galactic centre. A key prediction of this model is that the SMBH in A2261-BCG should be displaced from its centre. This would explain the offset of the outer isophotes with respect to the central region observed in the galaxies with the three largest cores (Postman et al. 2012; Dullo 2019; Gultekin et al. 2021).

Our model can also explain the bright 'knots' observed in the core region of A2261-BCG in one of two ways: either as the core of minor merging galaxies still on their way in or as a bound cluster of stars surrounding previously ejected SMBHs. The GW recoil model predicts a smaller and more tightly bound star cluster at the location of each knot than the 'minor merger' model that predicts a more extended and kinematically hotter distribution of stars (Merritt et al. 2009). Because HCSSs are predicted to have much lower luminosities than the knots in A2261-BGC (Merritt et al. 2009), we find the 'minor merger' scenario a more likely explanation for the knots.

We find that observed 'mass deficit' calculations in the literature are not reliable. These are typically calculated as the difference in stellar mass between the observed central surface brightness core and an inward extrapolation of an outer Sérsic profile fit. This is a reasonable assumption following a single major merger (in which a central core is formed through binary scouring alone). However, the calculation necessarily fails following subsequent mergers, both minor and major. Thus, the observed 'mass deficit' at the centres of giant elliptical galaxies, derived in the above way, cannot be directly used to constrain their merger histories.

Finally, we confirm with simulations that the cored surface brightness profiles produced by a sequence of dry galaxy mergers are in fact weak cusps in spatial density, and only appear cored in projection, in agreement with prior theoretical (Merritt \& Fridman 1995) and observational (Ferrarese et al. 1994; Lauer et al. 1995; Gebhardt et al. 1996) studies. Cores produced by GW recoil, on the other hand, result in genuinely flat 3D density profiles. Non-divergent central profiles are only observed for a few core galaxies (Lauer et al. 2002, 2005), and A2261-BCG is one of them (Postman et al. 2012), lending further support to our proposed scenario.

\section{ACKNOWLEDGEMENTS}

We thank Eugene Vasiliev for insightful discussions regarding the initial conditions for the galaxy models and Fani Dosopoulou for illu- 
minating discussions about the core properties of A2261-BCG. MD acknowledges support by ERC-Syg 810218 WHOLE SUN. FA acknowledges support from a Rutherford fellowship (ST/P00492X/1) from the Science and Technology Facilities Council.

\section{DATA AVAILABILITY}

The data underlying this article will be shared on reasonable request to the corresponding author.

\section{REFERENCES}

Antonini F., Merritt D., 2012, ApJ, 745, 83

Begelman M. C., Blandford R. D., Rees M. J., 1980, Nature, 287, 307

Behroozi P. S., Wechsler R. H., Conroy C., 2013, ApJ, 770, 57

Bekenstein J. D., 1973, ApJ, 183, 657

Blecha L. et al., 2016, MNRAS, 456, 961

Bonfini P., Graham A. W., 2016, ApJ, 829, 81

Boylan-Kolchin M., Ma C.-P., Quataert E., 2004, ApJ, 613, L37

Burke-Spolaor S., Gültekin K., Postman M., Lauer T. R., Taylor J. M., Lazio T. J. W., Moustakas L. A., 2017, ApJ, 849, 59

Caldwell N., Strader J., Romanowsky A. J., Brodie J. P., Moore B., Diemand J., Martizzi D., 2014, ApJ, 787, L11

Campanelli M., Lousto C. O., Marronetti P., Zlochower Y., 2006, Phys. Rev. Lett., 96, 111101

Campanelli M., Lousto C., Zlochower Y., Merritt D., 2007, ApJ, 659, L5

Carollo C. M., Franx M., Illingworth G. D., Forbes D. A., 1997, ApJ, 481, 710

Chandrasekhar S., 1943, ApJ, 97, 255

Chiaberge M., Tremblay G. R., Capetti A., Norman C., 2018, ApJ, 861, 56

De Lucia G., Blaizot J., 2007, MNRAS, 375, 2

Dehnen W., 2002, J. Comput. Phys., 179, 27

Dehnen W., 2005, MNRAS, 360, 892

Dehnen W., 2014, Comput. Astrophys. Cosmol., 1, 1

Devecchi B., Rasia E., Dotti M., Volonteri M., Colpi M., 2009, MNRAS, 394, 633

Dosopoulou F., Antonini F., 2017, ApJ, 840, 31

Dullo B. T., 2019, ApJ, 886, 80

Dullo B. T., Graham A. W., 2012, ApJ, 755, 163

Dullo B. T., Graham A. W., 2013, ApJ, 768, 36

Dullo B. T., Graham A. W., 2014, MNRAS, 444, 2700

Dullo B. T., Graham A. W., Knapen J. H., 2017, MNRAS, 471, 2321

Dutton A. A., Macciò A. V., 2014, MNRAS, 441, 3359

El-Zant A., Shlosman I., Hoffman Y., 2001, ApJ, 560, 636

Faber S. M. et al., 1997, AJ, 114, 1771

Feldmann R., Carollo C. M., Mayer L., 2011, ApJ, 736, 88

Ferrarese L., van den Bosch F. C., Ford H. C., Jaffe W., O’Connell R. W., 1994, AJ, 108, 1598

Gebhardt K. et al., 1996, AJ, 112, 105

Gerosa D., Sesana A., 2015, MNRAS, 446, 38

Goerdt T., Moore B., Read J. I., Stadel J., 2010, ApJ, 725, 1707

González J. A., Sperhake U., Brügmann B., Hannam M., Husa S., 2007, Phys. Rev. Lett., 98, 091101

Graham A. W., 2019, Publ. Astron. Soc. Aust., 36, e035

Graham A. W., Erwin P., Trujillo I., Asensio Ramos A., 2003, AJ, 125, 2951

Gualandris A., Merritt D., 2008, ApJ, 678, 780

Gualandris A., Merritt D., 2012, ApJ, 744, 74

Gultekin K., Burke-Spolaor S., Lauer T. R., Lazio T. J. W., Moustakas L. A., Ogle P., Postman M., 2021, ApJ, 906, 48

Healy J., Lousto C. O., Zlochower Y., 2014, Phys. Rev. D, 90, 104004

Healy J., Lousto C. O., Ruchlin I., Zlochower Y., 2018, Phys. Rev. D, 97, 104026

Hills J. G., 1983, AJ, 88, 1269
Hoffman L., Loeb A., 2007, MNRAS, 377, 957

Iwasawa M., Funato Y., Makino J., 2006, ApJ, 651, 1059

Iwasawa M., Funato Y., Makino J., 2008, preprint (arXiv:0801.0859)

Johansson P. H., Naab T., Burkert A., 2009, ApJ, 690, 802

Kalfountzou E., Santos Lleo M., Trichas M., 2017, ApJ, 851, L15

Khochfar S., Burkert A., 2006, A\&A, 445, 403

King I. R., Minkowski R., 1966, ApJ, 143, 1002

King A., Nealon R., 2019, MNRAS, 487, 4827

Komossa S., 2012, Adv. Astron., 2012, 364973

Kormendy J., Bender R., 1996, ApJ, 464, L119

Krajnović D. et al., 2013, MNRAS, 433, 2812

Kravtsov A. V., 2013, ApJ, 764, L31

Kulkarni G., Loeb A., 2012, MNRAS, 422, 1306

Lauer T. R. et al., 1995, AJ, 110, 2622

Lauer T. R. et al., 2002, AJ, 124, 1975

Lauer T. R. et al., 2005, AJ, 129, 2138

Lauer T. R. et al., 2007, ApJ, 662, 808

Li Y., Mo H. J., van den Bosch F. C., Lin W. P., 2007, MNRAS, 379, 689

Lousto C. O., Healy J., 2019, Phys. Rev. D, 100, 104039

Lousto C. O., Zlochower Y., 2011, Phys. Rev. Lett., 107, 231102

Lousto C. O., Zlochower Y., Dotti M., Volonteri M., 2012, Phys. Rev. D, 85, 084015

Márquez I., Lima Neto G. B., Capelato H., Durret F., Gerbal D., 2000, A\&A, 353,873

Martizzi D., Teyssier R., Moore B., 2012, MNRAS, 420, 2859

Mehrgan K., Thomas J., Saglia R., Mazzalay X., Erwin P., Bender R., Kluge M., Fabricius M., 2019, ApJ, 887, 195

Merritt D., 2006, ApJ, 648, 976

Merritt D., 2013, Dynamics and Evolution of Galactic Nuclei. Princeton University Press, Princeton

Merritt D., Fridman T., 1995, in Buzzoni A., Renzini A., Serrano A., eds, ASP Conf. Ser. Vol. 86, Fresh Views of Elliptical Galaxies. Astron. Soc. Pac., San Francisco, p. 13

Merritt D., Milosavljević M., Favata M., Hughes S. A., Holz D. E., 2004, ApJ, 607, L9

Merritt D., Schnittman J. D., Komossa S., 2009, ApJ, 699, 1690

Milosavljević M., Merritt D., 2001, ApJ, 563, 34

Milosavljević M., Merritt D., Rest A., van den Bosch F. C., 2002, MNRAS, 331, L51

Moster B. P., Naab T., White S. D. M., 2013, MNRAS, 428, 3121

Naab T., Ostriker J. P., 2017, ARA\&A, 55, 59

Naab T., Trujillo I., 2006, MNRAS, 369, 625

Naab T., Johansson P. H., Ostriker J. P., 2009, ApJ, 699, L178

Nasim I., Gualandris A., Read J., Dehnen W., Delorme M., Antonini F., 2020, MNRAS, 497, 739

Nasim I., Petrovich C., Nasim A., Dosopoulou F., Antonini F., 2021, MNRAS, preprint (arXiv:2101.07266)

Navarro J. F., Frenk C. S., White S. D. M., 1996, ApJ, 462, 563

Oliva-Altamirano P. et al., 2015, MNRAS, 449, 3347

Peters P. C., 1964, Phys. Rev., 136, 1224

Pontzen A., Governato F., 2012, MNRAS, 421, 3464

Postman M. et al., 2012, ApJ, 756, 159

Power C., Navarro J. F., Jenkins A., Frenk C. S., White S. D. M., Springel V., Stadel J., Quinn T., 2003, MNRAS, 338, 14

Quinlan G. D., 1996, New Astron., 1, 35

Rantala A., Johansson P. H., Naab T., Thomas J., Frigo M., 2018, ApJ, 864, 113

Rantala A., Johansson P. H., Naab T., Thomas J., Frigo M., 2019, ApJ, 872, L17

Read J. I., Gilmore G., 2005, MNRAS, 356, 107

Read J. I., Goerdt T., Moore B., Pontzen A. P., Stadel J., Lake G., 2006, MNRAS, 373, 1451

Read J. I., Walker M. G., Steger P., 2019, MNRAS, 484, 1401

Sérsic J. L., 1963, Boletin de la Asociacion Argentina de Astronomia La Plata Argentina, 6, 41

Sérsic J. L., 1968, Atlas de Galaxias Australes. Observatorio Astronomico, Cordoba, Argentina 
Sesana A., Haardt F., Madau P., 2006, ApJ, 651, 392

Terzić B., Graham A. W., 2005, MNRAS, 362, 197

Thomas J., Ma C.-P., McConnell N. J., Greene J. E., Blakeslee J. P., Janish R., 2016, Nature, 532, 340

Toomre A., 1977, in Tinsley B. M., Larson Richard B., Gehret D. C., eds, Evolution of Galaxies and Stellar Populations. p. 401

Trujillo I., Erwin P., Asensio Ramos A., Graham A. W., 2004, AJ, 127, 1917

Vasiliev E., 2019, MNRAS, 482, 1525
Von Der Linden A., Best P. N., Kauffmann G., White S. D. M., 2007, MNRAS, 379, 867

White S. D. M., Rees M. J., 1978, MNRAS, 183, 341

Zubovas K., King A., 2019, MNRAS, 489, 1373

This paper has been typeset from a $\mathrm{T}_{\mathrm{E}} \mathrm{X} / \mathrm{LT} \mathrm{E} \mathrm{X}$ file prepared by the author. 